\title{
SIC-Coding Schemes for Underlay Two-Way Relaying Cognitive Networks
}

\author{
Pham Ngoc Son $\mathbb{D}^{1},{ }^{1}$ Tran Trung Duy, ${ }^{2}$ and Khuong Ho-Van ${ }^{3,4}$ \\ ${ }^{1}$ Ho Chi Minh City University of Technology and Education, Ho Chi Minh City, Vietnam \\ ${ }^{2}$ Posts and Telecommunications Institute of Technology, Ho Chi Minh City, Vietnam \\ ${ }^{3}$ Ho Chi Minh City University of Technology (HCMUT), Ho Chi Minh City, Vietnam \\ ${ }^{4}$ Vietnam National University Ho Chi Minh City, Ho Chi Minh City, Vietnam \\ Correspondence should be addressed to Pham Ngoc Son; sonpndtvt@hcmute.edu.vn
}

Received 22 March 2020; Revised 14 July 2020; Accepted 30 July 2020; Published 24 August 2020

Academic Editor: Carlos T. Calafate

Copyright (C) 2020 Pham Ngoc Son et al. This is an open access article distributed under the Creative Commons Attribution License, which permits unrestricted use, distribution, and reproduction in any medium, provided the original work is properly cited.

\begin{abstract}
In this paper, we propose an underlay two-way relaying scheme with the successive interference cancellation (SIC) solution in which two secondary sources transmit simultaneously their data to each other through secondary relays. The proposed scheme is operated in only two time slots and under an interference constraint of a primary receiver, denoted as the UTW-2TS scheme. In the UTW-2TS scheme, the secondary relays employ the SIC operation to decode successively the data from received broadcast signals and then encode these data by two techniques: digital network coding (DNC) enforced by XOR operations (denoted as the UTW-2TS-DNC protocol) and superposition coding (SC) enforced by power domain additions (denoted as the UTW-2TS-SC protocol). A selected secondary relay which subjects to maximize decoding capacities and to minimize collection time of channel state information in two protocols UTW-2TS-DNC and UTW-2TS-SC experiences residual interferences from imperfect SIC operations. Outage probabilities and throughputs are solved in terms of exact closed-form expressions to evaluate the system performance of the proposed protocols. Simulation and analysis results provide performance enhancement of the proposed protocols UTW-2TS-DNC and UTW-2TS-SC owing to increase the number of the cooperative secondary relays, the interference constraints, and the distances from the secondary network to the primary receiver. The best throughputs are pointed at optimal interference power allocation coefficients and optimal locations of the selected secondary relay. Considering the same power consumption, the UTW-2TS-DNC protocol outperforms the UTW-2TS-SC protocol. Finally, the simulation results are collected to confirm the exact analysis values of the outage probabilities and throughputs.
\end{abstract}

\section{Introduction}

In recent years, the radio spectrum has become scarce due to the increasing bandwidth demand for mobile multimedia services and the explosive development of next-generation wireless networks such as wireless sensor networks, Internet of Things (IoT), and fifth-generation (5G) networks. On the other hand, the utilization of the licensed frequency spectrum versus time and space is low [1]. In this context, cognitive radio was proposed as an effective spectrum sharing solution in which secondary users (SUs) coexist with primary users (PUs) [2]. The SUs can operate flexibly and intelligently in (interweave, overlay, and underlay) protocols to access the licensed frequency spectra of the PUs as long as quality of service $(\mathrm{Q} o S)$ of the primary network is maintained $[3,4]$. In the interweave cognitive radio [5], the SUs use spectrum sensing methods to detect the unoccupied spectra to avoid interference to the PUs. In an opposite way, the SUs in the overlay and underlay methods can access the licensed bands at the same time with the PUs [6-9]. The overlay approach requires the cooperation between the SUs and the PUs; i.e., the secondary transmitters have to combine the data of the SUs and the PUs and then send the combined data to the intended secondary and primary receivers [6]. The SUs in the underlay approach can access the spectra at any time provided that the interferences affected on the PUs must be below a tolerable interference level [7-9]. Based on the required QoS, the PUs can calculate the tolerable interference 
level and send it to the secondary network so that the SUs can appropriately adjust their transmit powers.

Two-way communication networks have been much interested in switching data between interactive users in which many research studies have been launched to enhance performance [10]. Two-way cooperation solutions investigated in [10] have great attractions owing to increase significantly spectrum utilization efficiency. The operation of two-way relaying is to exchange data between users with the help of other intermediate users as amplify-and-forward (AF) and decode-and-forward (DF) devices, denoted as relays. In [11], an optimum relay is selected to decode received signals from the transmit users, mix these data by XOR operation, and then broadcast back to both users. The operation of the optimum relay in [11] is named as digital network coding (DNC).

1.1. Related Work and Motivation. Proposals for increasing performance in the underlay two-way relaying networks have been studied in [12-18]. The authors in [12] combined DNC and opportunistic relay selection (ORS) to decrease the outage performance of the secondary two-way relaying network under the interference constraint required by the primary receiver. Moreover, the best relay selection strategy in the third time slot, which follows a max-min criterion, was proposed in [12] to significantly decrease the system outage probabilities, as compared with the traditional DNC approach [12]. By expanding the published work [12], Toan et al. in $[13,14]$ analyzed the performance of the underlay two-way relaying communication systems with the presence of multiple primary receivers. The authors in [15] evaluated the outage probabilities and symbol error probabilities of many primary one-way networks and a secondary two-way network operating on both the independent and identically distributed (i.i.d.) and independent but nonidentically distributed (i.n.i.d.) Nakagami- $m$ fading channels. Imperfect channel state information (CSI) from the SUs to the PUs have been included in probability analyses [16]. The authors exploited both the direct and relaying links with appropriate diversity combinations in an underlay threephase two-way scheme [17]. In [18], the authors considered an underlay wiretap cognitive two-way relaying network in which two SUs exchange their messages via multiple secondary DF relays in the presence of an eavesdropper. In [18], DNC, ORS, and artificial noises were used to mitigate eavesdropping attacks. However, above investigations perform on three orthogonal time slots, and hence, the bandwidth utilization efficiency is only $2 / 3$ (two data per three time slots). This motivates us to propose new two-way relaying approaches for the underlay cognitive radio network, where only two time slots are used to obtain the data rate of $2 / 2$.

In [19], the authors applied successive interference cancellation (SIC) technique to sequentially decode signals received from multiple sources. With SIC, the spectral efficiency of the two-way relaying systems is improved because the number of time slots decreases. Indeed, SIC is one of the core technologies in nonorthogonal multiple access (NOMA) systems which can assign nonorthogonal resources such as power, time, and code to different users [20]. For the NOMA systems operating in the power domain, multiple transmit signals that are allocated with different transmit powers are merged by the superposition coding (SC) before they are sent to intended receivers. At each receiver, the signal with higher transmit power is first decoded, and it is then removed from the received signal. This process, named SIC, is repeated until the receiver obtains the desired signal [21]. The authors in [19] pointed out some implementation problems of the SIC utilization such as complexity and residual interference after performing interference cancellations. These issues will lead to lower decoding capacities than expected ones. In [22], the authors evaluated two-way relaying NOMA systems fully in terms of the outage probability, outage floor, ergodic capacity, and power allocation. The system performance of the proposed protocol in [22] outperforms that of three-time slot two-way relaying systems with orthogonal multiple access. In addition, we have applied the SIC scheme with DNC and ORS at the last time slot (the second time slot) for traditional two-way relaying networks in [23]. The results obtained in [23] presented improvements of the system performances as well as bandwidth utilization efficiencies, as compared with the corresponding scenarios without using SIC. However, the previous published works [19, 22, 23] only considered the conventional wireless networks; i.e., the underlay cognitive network was not studied.

1.2. Contributions. Motivated by above issues, in this paper, we propose an underlay two-way relaying scheme with the SIC solution in which two secondary sources send concurrently their data to each other through multiple secondary relays under an interference constraint of a primary receiver. The proposed scheme is operated in two time slots, denoted as the UTW-2TS scheme. In the UTW-2TS scheme, the secondary relays employ the SIC operation to decode successively the data from received broadcast signals. The data carried by the stronger channel gains is decoded firstly and will be subtracted to detect the remaining data. These decoded data are encoded by two techniques: the DNC enforced by XOR operations (denoted as the UTW-2TSDNC protocol) and the SC enforced by power domain additions (denoted as the UTW-2TS-SC protocol). In addition, because of imperfect operations of the SIC, the secondary relays experience residual interferences. A secondary relay is selected in two protocols UTW-2TS-DNC and UTW2TS-SC which subjects to maximize decoding capacities and to minimize collection time of CSI. Outage probabilities and throughputs are solved in terms of exact closed-form expressions to evaluate the system performance of the proposed UTW-2TS-DNC and UTW-2TS-SC protocols. These outage probability and throughput expressions are proved by doing the Monte Carlo simulations. The contributions of this paper are cataloged as follows:

(1) Proposing SIC-based underlay two-way relaying cognitive protocols which enhance the data rate, as compared with the corresponding ones without using the SIC 
(2) Exactly analyzing the outage probabilities and throughputs of two secondary sources in two operation protocols UTW-2TS-DNC and UTW-2TS-SC with the same two-way relaying model in which the SIC solution in the selected secondary relay is practically considered in the perfect and imperfect cases

(3) The UTW-2TS-DNC protocol outperforms the UTW-2TS-SC protocol with the same power consumption

(4) The proposed UTW-2TS-DNC and UTW-2TS-SC protocols can achieve the best throughputs at optimal interference power allocation coefficients and optimal locations of the selected secondary relay. In addition, we examine the throughputs of the UTW-2TSSC protocol versus the changes of the power allocation coefficients

(5) The system performances in terms of the outage probabilities and throughputs are improved when the number of the cooperative secondary relays, the interference constraints, and the distances from the secondary nodes to the primary receiver are increased as well as when the residual interference powers decrease

1.3. Paper Organization and Notations. This paper is organized into sections as follows. Section 2 presents a system model of an underlay two-way relaying scheme with multiple secondary relays. Section 3 analyzes outage probabilities and throughputs of the proposed protocols UTW-2TS-DNC and UTW-2TS-SC. Results and discussions are shown in Section 4. Lastly, Section 5 summarizes contributions.

The notations used in this paper are denoted as follows: $f_{Y}(\cdot)$ and $F_{Y}(\cdot)$ denote, respectively, the probability density function (PDF) and the cumulative distribution function (CDF) of a random variable (RV) $Y$; $\operatorname{Pr}\{\Xi\}$ denotes the probability operation of an event $\Xi ; E\{\cdot\}$ denotes the expectation operator; $\left(\begin{array}{l}p \\ M\end{array}\right)$ denotes the binomial coefficient $\left(\left(\begin{array}{c}p \\ M\end{array}\right)=M ! / p !(M-p) !\right) ; \oplus$ denotes XOR math operation; and $\Gamma[u, v]$ is the upper incomplete Gamma function.

\section{System Model}

Figure 1 presents a system model of an underlay two-way relaying scheme. In this scheme, secondary sources $S_{1}$ and $S_{2}$ own data $x_{1}$ and $x_{2}\left(x_{1} \in S_{1}\right.$ and $\left.x_{2} \in S_{2}\right)$, respectively. The exchange of information is achieved between the $S_{1}$ and the $S_{2}$ through a cluster of $M$ intermediate secondary relays $R_{i}$, where $i=\{1,2, \cdots, M\}$ [12]. More specifically, the data $x_{1}$ of $S_{1}$ is exchanged with the data $x_{2}$ of $S_{2}$. The secondary network including $S_{1}, S_{2}$, and $R_{i}$ suffers an interference constraint of a primary receiver (PR), denoted as $I$. The secondary relays can perform the SIC technique to decode the

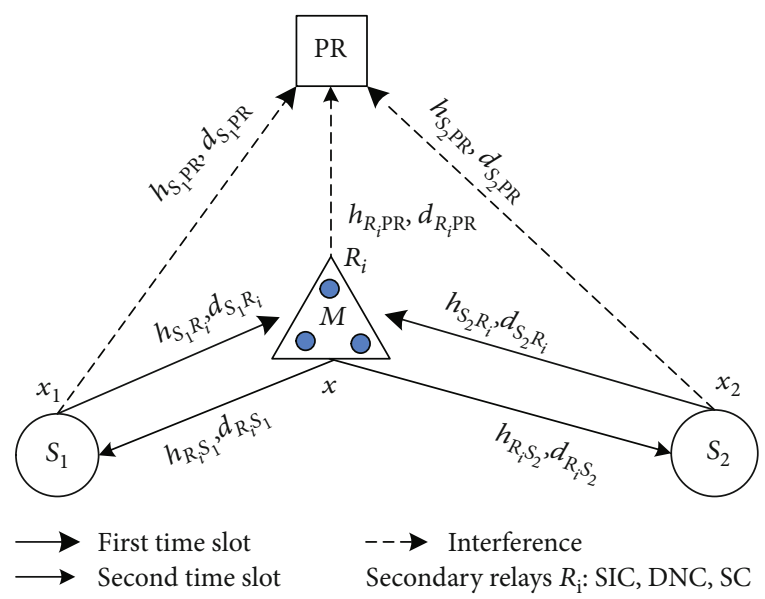

Figure 1: System model of an underlay two-way relaying scheme.

received data-carried signals successively in an interval of a time slot.

We assume that a direct link between the secondary sources $S_{1}$ and $S_{2}$ does not exist due to the far distance or deep shadow fading $[18,23,24]$. It is also assumed that the secondary and primary nodes are installed with a single antenna, and additive noises at all the receivers are zeromean Gaussian random variables (RVs) whose mean is zero, and variance is $\sigma^{2}$.

In Figure 1, $h_{X Y}$ and $d_{X Y}$ denote the flat-block Rayleigh fading channel coefficient and the normalized link distance of link $X-Y$, respectively, where $X, Y \in\left\{S_{1}, S_{2}, R_{i}, \mathrm{PR}\right\}$. Because the secondary relays $R_{i}$ are located in the cluster, where the secondary relays $R_{i}$ are close to each other, the normalized distances can be assumed to be identical, i.e., $d_{S_{1} R_{i}}=d_{R_{i} S_{1}}=d_{1}, \quad d_{S_{2} R_{i}}=d_{R_{i} S_{2}}=d_{2}, \quad d_{R_{i} \mathrm{PR}}=d_{3}, \quad d_{S_{1} \mathrm{PR}}=d_{4}$, and $d_{S_{2} \mathrm{PR}}=d_{5}[25,26]$. Because $\left|h_{X Y}\right|$ have Rayleigh distributions, the channel gains $g_{X Y}=\left|h_{X Y}\right|^{2}$ are exponential RVs with the PDFs $f_{g_{X Y}}(y)=\lambda_{X Y} e^{-\lambda_{X Y} y}$ and the CDFs $F_{g_{X Y}}(y)=$ $1-e^{-\lambda_{X Y} y}$, where $\lambda_{X Y}=d_{X Y}^{\eta}$, and $\eta$ is a path loss exponent (see $[23,27,28])$. Moreover, we have $\lambda_{S_{1} R_{i}}=\lambda_{R_{i} S_{1}}=\lambda_{1}, \lambda_{S_{2} R_{i}}$ $=\lambda_{R_{i} S_{2}}=\lambda_{2}, \lambda_{R_{i} \mathrm{PR}}=\lambda_{3}, \lambda_{S_{1} \mathrm{PR}}=\lambda_{4}$, and $\lambda_{S_{2} \mathrm{PR}}=\lambda_{5}$.

In the underlay cognitive radio network, the secondary network coexisted in the same frequency with the primary network and satisfies that the interference power at the primary receiver $(\mathrm{PR})$ is less than or equal the constraint $I[29,30]$. We have the inequality

$$
\left\{\begin{array}{l}
P_{S_{1}} g_{S_{1} \mathrm{PR}}+P_{S_{2}} g_{S_{2} \mathrm{PR}} \leq I \\
P_{R_{i}} g_{R_{i} \mathrm{PR}} \leq I, \quad i=\{1,2, \cdots, M\}
\end{array}\right.
$$

where $P_{S_{1}}, P_{S_{2}}$, and $P_{R_{i}}$ are transmit powers of the secondary source $S_{1}$, the secondary source $S_{2}$, and the secondary relays $\mathrm{R}_{i}$, respectively.

From (1), the transmit powers of the secondary transmitters can be set as $P_{S_{1}}=\alpha_{1} I / g_{S_{1} \mathrm{PR}}, P_{S_{2}}=\alpha_{2} I / g_{S_{2} \mathrm{PR}}$, and $P_{R_{i}}=$ $I / g_{R_{i} \mathrm{PR}}$, where $\alpha_{1}$ and $\alpha_{2}$ are interference power allocation coefficients, $0<\alpha_{1}, \alpha_{2}<1$, and $\alpha_{1}+\alpha_{2}=1$. 
Comment 1. In practice, the transmit powers $P_{X}$ must be below a maximum power (denoted by $P_{X, \max }$ ), where $X \in$ $\left\{S_{1}, S_{2}, R_{i}\right\}$. Therefore, $P_{X}$ should be formulated as $P_{X}=$ $\min \left(P_{X, \max }, u I / g_{X P R}\right)$, where $u \in\left\{\alpha_{1}, \alpha_{2}, 1\right\}$. Suppose that $P_{X \text {, max }} \gg I$ (e.g., the $X$ nodes are near the PR node); hence, we can approximate $P_{X}$ by $P_{X} \approx u I / g_{X P R}$. It is worth noting that this assumption is used in many published literatures, i.e., $[12,26,30-36]$.

The operation principle of the UTW-2TS schemes happens in two time slots as follows. In the first time slot, secondary sources $S_{1}$ and $S_{2}$ transmit simultaneously the data-carried signals $x_{1}$ and $x_{2}$, respectively, to all secondary relays under interference constraint of the primary receiver $(\mathrm{PR})$. A selected secondary relay, denoted as $R_{m}$, where $m=\{1,2, \cdots, M\}$, decodes the desired data by applying the SIC process. In the second time slot (last time slot), the $R_{m}$ creates the new data $x$ by the DNC technique, denoted as the UTW-2TS-DNC protocol, or by the SC technique, denoted as the UTW-2TS-SC protocol. The new data will be transmitted back to two secondary sources $S_{1}$ and $S_{2}$. Collection of CSI and system parameters for decoding data, cancelling interferences, subtracting selfinterference components, and selecting the cooperative relay are performed by the medium access control (MAC) protocol as specified in [37].

The secondary sources $S_{1}$ and $S_{2}$ transmit simultaneously the data $x_{1}$ and $x_{2}$, respectively, to the secondary relays $R_{i}$ at the same time (the first time slot) and the same frequency as the uplink NOMA operation, where $i=\{1,2, \cdots, M\}$. The received signal at the $R_{i}$ is expressed as

$$
y_{R_{i}}=\sqrt{P_{S_{1}}} x_{1} h_{S_{1} R_{i}}+\sqrt{P_{S_{2}}} x_{2} h_{S_{2} R_{i}}+n_{R_{i}}
$$

where $E\left\{\left|x_{1}\right|^{2}\right\}=E\left\{\left|x_{2}\right|^{2}\right\}=1$, and $n_{R_{i}}$ presents the Gaussian noises at the secondary relays $R_{i}$ with the same variance $\sigma^{2}$.

The secondary relays $R_{i}$ can compute the distances $d_{S_{1} R_{i}}$ and $d_{S_{2} R_{i}}$ by taking coordinate parameters in received request-to-send (RTS) messages of the secondary sources $S_{1}$ and $S_{2}$ in the setup phase [37]. Coordinates of the nodes can be received from the navigation systems. Hence, the secondary relays $R_{i}$ can make decoding decisions of the signals $x_{1}$ and $x_{2}$ in (2) by the SIC technique as follows.

Case 1. $\left(d_{S_{1} R_{i}} \leq d_{S_{2} R_{i}}\right.$ or $E\left\{g_{S_{1} R_{i}}\right\} \geq E\left\{g_{S_{2} R_{i}}\right\}\left(R_{i}\right.$ is closer $S_{1}$ than $\left.S_{2}\right)$ ).

In this case, the data $x_{1}$ in (2) is decoded by the secondary relay $R_{i}$ firstly whereas a signal $\sqrt{P_{S_{2}}} x_{2} h_{S_{2} R_{i}}$ containing $x_{2}$ is treated as interference. The received signal-to-interferenceplus-noise ratios (SINRs) at the $R_{i}$ to decode $x_{1}$ are obtained from (2) as

$$
\gamma_{R_{i} \rightarrow x_{1}}^{(1)}=\frac{P_{S_{1}} g_{S_{1} R_{i}}}{P_{S_{2}} g_{S_{2} R_{i}}+\sigma^{2}}=\frac{\alpha_{1} Q g_{S_{1} R_{i}} g_{S_{2} P R}}{\alpha_{2} Q g_{S_{2} R_{i}} g_{S_{1} \mathrm{PR}}+g_{S_{1} \mathrm{PR}} g_{S_{2} \mathrm{PR}}}
$$

where $Q=I / \sigma^{2}$.
In (3), to increase the decoding capacity for the data $x_{1}$, decrease the collection of the CSI, and minimize help of the cooperative secondary relays, we only select one secondary relay among $M$ secondary relays. Indeed, the selected secondary relay $R_{m}$ is obtained as $R_{m}=\arg \max _{i=1,2, \cdots, M} g_{S_{1} R_{i}}$. Then, the CDF and PDF of the RV $g_{S_{1} R_{m}}$ are correspondingly expressed as ([28], eqs. 7-14)

$$
\begin{aligned}
& F_{g_{S_{1} R_{m}}}(y)=\left(1-e^{-\lambda_{1} y}\right)^{M}=\sum_{p=0}^{M}\left(\begin{array}{c}
p \\
M
\end{array}\right)(-1)^{p} e^{-p \lambda_{1} y}, \\
& f_{g_{S_{1} R_{m}}}(y)=M \lambda_{1} e^{-\lambda_{1} y}\left(1-e^{-\lambda_{1} y}\right)^{M-1} .
\end{aligned}
$$

After decoding $x_{1}$ successfully, the inference component $\sqrt{P_{S_{1}}} x_{1} h_{S_{1} R_{m}}$ in (2) can be cancelled completely or partly by the SIC technique in which the transmit power $P_{S_{1}}$ and the channel coefficient $h_{S_{1} R_{m}}$ were known in the setup phase [37]. The remaining received SINR at the selected secondary relay $R_{m}$ to decode $x_{2}$ is inferred as

$$
\gamma_{R_{m} \rightarrow x_{2}}^{(1)}=\frac{P_{S_{2}} g_{S_{2} R_{m}}}{\varepsilon I\left|r_{m}\right|^{2}+\sigma^{2}},
$$

where $r_{m}$ is a residual interference part at the secondary relay $R_{m}$ due to the imperfect SIC operations and can be modeled as an identical complex normal distribution $r_{m} \sim \mathrm{CN}(0, \Psi)$ [19] with zero mean and same variance $\Psi$ ( $\Psi$ is also the power of the residual interference part), and hence, $g_{m}=$ $\left|r_{m}\right|^{2}$ are also exponentially distributed RVs with the PDF as $f_{g_{m}}(y)=\Omega e^{-\Omega y}$ and the CDF as $F_{g_{m}}(y)=1-e^{-\Omega y}$ ([28], eqs. 6-68), where $\Omega=1 / \Psi ; \varepsilon=0$ and $\varepsilon=1$ denote perfect and imperfect interference cancellation at the secondary relay $R_{m}$, respectively.

Substituting $P_{S_{2}}=\alpha_{2} I / g_{S_{2} \mathrm{PR}} \quad$ into (6), $\quad \gamma_{R_{m} \rightarrow x_{2}}^{(1)}$ is expressed as

$$
\gamma_{R_{m} \rightarrow x_{2}}^{(1)}=\frac{\alpha_{2} Q g_{S_{2} R_{m}}}{g_{S_{2} \mathrm{PR}}\left(\varepsilon Q g_{m}+1\right)} .
$$

Case 2. $\left(d_{S_{1} R_{i}}>d_{S_{2} R_{i}}\right.$ or $E\left\{g_{S_{1} R_{i}}\right\}<E\left\{g_{S_{2} R_{i}}\right\}\left(R_{i}\right.$ is closer $S_{2}$ than $\left.S_{1}\right)$ ).

Similarly, the secondary relay $R_{i}$ decodes the data $x_{2}$ firstly with the interference signal $\sqrt{P_{S_{1}}} x_{1} h_{S_{1} R_{i}}$, where $i=$ $\{1,2, \cdots, M\}$. The SINRs at the $R_{i}$ for decoding $x_{2}$ and $x_{1}$ are expressed, respectively, as

$$
\begin{aligned}
& \gamma_{R_{i} \rightarrow x_{2}}^{(2)}=\frac{P_{S_{2}} g_{S_{2} R_{i}}}{P_{S_{1}} g_{S_{1} R_{i}}+\sigma^{2}}=\frac{\alpha_{2} Q g_{S_{2} R_{i}} g_{S_{1} \mathrm{PR}}}{\alpha_{1} Q g_{S_{1} R_{i}} g_{S_{2} \mathrm{PR}}+g_{S_{1} \mathrm{PR}} g_{S_{2} \mathrm{PR}}}, \\
& \gamma_{R_{i} \rightarrow x_{1}}^{(2)}=\frac{P_{S_{1}} g_{S_{1} R_{i}}}{\varepsilon I g_{i}+\sigma^{2}}=\frac{\alpha_{1} Q g_{S_{1} R_{i}}}{g_{S_{1} \mathrm{PR}}\left(\varepsilon Q g_{i}+1\right)} .
\end{aligned}
$$


In the second time slot, if the selected secondary relay $R_{m}$ decodes successfully both data $x_{1}$ and $x_{2}$ in the first time slot, the $R_{m}$ creates a new data $x$ as

$x=\left\{\begin{array}{l}x_{1} \oplus x_{2}, \quad \text { digital network coding }(\text { the UTW-2TS-DNC protocol }), \\ \sqrt{\beta_{1}} x_{1}+\sqrt{\beta_{2}} x_{2}, \quad \text { superposition coding }(\text { the UTW-2TS-SC protocol), }\end{array}\right.$

where $\beta_{1}$ and $\beta_{2}$ are power allocation coefficients to data $x_{1}$ and $x_{2}$, respectively, and satisfy conditions as [21]

$$
\left\{\begin{array}{l}
\beta_{1}+\beta_{2}=1, \quad 0 \leq \beta_{1} \leq 1,0 \leq \beta_{2} \leq 1, \\
\beta_{1} \geq \beta_{2}, \quad d_{S_{1} R_{m}} \leq d_{S_{2} R_{m}}(\text { Case } 1), \\
\beta_{1}<\beta_{2}, \quad d_{S_{1} R_{m}}>d_{S_{2} R_{m}}(\text { Case } 2) .
\end{array}\right.
$$

We remark that for the UTW-2TS-SC protocol as in (10) and (11), the selected secondary relay $R_{m}$ performs the downlink NOMA operation to allocate powers to data $x_{1}$ and $x_{2}$ as $x=\sqrt{\beta_{1}} x_{1}+\sqrt{\beta_{2}} x_{2}$ in which $x_{1}$ and $x_{2}$ are the desired data of the secondary sources $S_{2}$ and $S_{1}$, respectively [21]. If the $S_{k}$ is closer to the $R_{m}$, the $R_{m}$ will allocate the lower power for the data $x_{l}$, where $k, l=\{1,2\}$ and $l \neq k$ and vice versa. More specifically, in Case $1\left(d_{S_{1} R_{m}} \leq d_{S_{2} R_{m}}\right)$, the $S_{1}$ is the nearby user and the $S_{2}$ is the distant user. Hence, the $R_{m}$ uses the lower power allocation coefficient $\beta_{2}\left(\beta_{2} \leq \beta_{1}\right)$ to transmit the data $x_{2}$ to the $S_{1}$ and the higher power allocation coefficient $\beta_{1}$ to transmit the data $x_{1}$ to the $S_{2}$. In addition, we note that the secondary relay $R_{m}$ in Case 2 is selected by $\mathrm{R}_{m}=\arg \max _{i=1,2, \cdots, M} \mathrm{~g}_{\mathrm{S}_{2} \mathrm{R}_{i}}$.

The coded data $x$ in (10) will be broadcasted back to two secondary sources $S_{1}$ and $S_{2}$, and then the received signals at the secondary sources $S_{k}$ are expressed as

$$
\begin{aligned}
y_{S_{k}} & =\sqrt{P_{R_{m}}} x h_{R_{m} S_{k}}+n_{S_{k}} \\
& =\left\{\begin{array}{l}
\sqrt{P_{R_{m}}} h_{R_{m} S_{k}}\left(x_{1} \oplus x_{2}\right)+n_{S_{k}}(\text { the UTW-2TS-DNC protocol) } \\
\sqrt{P_{R_{m}}} h_{R_{m} S_{k}}\left(\sqrt{\beta_{1}} x_{1}+\sqrt{\beta_{2}} x_{2}\right)+n_{S_{k}}(\text { the UTW-2TS-SC protocol) }
\end{array}\right. \\
& =\left\{\begin{array}{l}
\sqrt{P_{R_{m}}} h_{R_{m} S_{k}}\left(x_{1} \oplus x_{2}\right)+n_{S_{k}}(\text { the UTW-2TS-DNC protocol) } \\
\sqrt{\beta_{l} P_{R_{m}}} h_{R_{m}} S_{k} x_{l}+\sqrt{\beta_{k} P_{R_{m}}} h_{R_{m} S_{k}} x_{k}+n_{S_{k}} \text { (the UTW-2TS-SC protocol). }
\end{array}\right.
\end{aligned}
$$

where $n_{S_{k}}$ presents the Gaussian noises at the secondary sources $S_{k}$ with the same variance $\sigma^{2}$.

For the UTW-2TS-DNC protocol with $x=x_{1} \oplus x_{2}$ in (12), the received SINRs at the secondary sources $S_{k}$ can be obtained from (12) to take $x$ as

$$
\gamma_{S_{k} \rightarrow x}^{\mathrm{UTW}-2 \mathrm{TS}-\mathrm{DNC}}=\frac{P_{R_{m}}\left|h_{R_{m} S_{k}}\right|^{2}}{\sigma^{2}}=\frac{Q g_{R_{m} S_{k}}}{g_{R_{m} P R}} .
$$

The decoding method of the secondary sources $S_{k}$ to take the desired data is performed by decoding operations of the network coding; i.e., the $S_{1}$ takes the data $x_{2}$ by XOR opera- tions of its data $x_{1}$ with the compressed data $x$ as $x_{1} \oplus x=$ $x_{1} \oplus x_{1} \oplus x_{2}=x_{2}$.

For the UTW-2TS-SC protocol, the received signals at the secondary sources $S_{k}$ are shown more clearly as

$$
y_{S_{k}}=\underbrace{\sqrt{\beta_{l} P_{R_{m}}} h_{R_{m} S_{k}} x_{l}}_{\text {desired component }}+\underbrace{\sqrt{\beta_{k} P_{R_{m}}} h_{R_{m} S_{k}} x_{k}}_{\text {self-interference component }}+n_{S_{k}} .
$$

In (14), the secondary source $S_{k}$ owns the data $x_{k}$ and does not decode this data. In addition, the $S_{k}$ can estimate the fading channel coefficient $h_{R_{m} S_{k}}$ from receiving the setup messages of the selected secondary relay $R_{m}$ and takes the system parameters as the transmit power $P_{R_{m}}$ and the power allocation coefficient $\beta_{k}$ during the setup time, where $k=\{1,2\}$, [37]. Hence, the $S_{k}$ knows the parameters $x_{k}, h_{R_{m} S_{k}}$, $P_{R_{m}}$, and $\beta_{k}$, which then can cancel out the self-interference component $\sqrt{\beta_{k} P_{R_{m}}} h_{R_{m} s_{k}} x_{k}$ in (14) $[38,39]$. The received signals at the $S_{k}$ after subtracting the known self-interference components are obtained as

$$
\widehat{y_{S_{k}}}=\underbrace{\sqrt{\beta_{l} P_{R_{m}}} h_{R_{m} S_{k}} x_{l}}_{\text {desired component }}+n_{S_{k}}
$$

The received SINRs at the secondary sources $S_{k}$ can be computed to take the desired data $x_{l}$ as

$$
\gamma_{S_{k} \rightarrow x_{l}}^{\mathrm{UTW}-2 \mathrm{TS}-\mathrm{SC}}=\frac{P_{R_{m}} \beta_{l}\left|h_{R_{m} S_{k}}\right|^{2}}{\sigma^{2}}=\frac{Q \beta_{l} g_{R_{m} S_{k}}}{g_{R_{m} P R}} .
$$

\section{Outage Probability and Throughput Analyses}

In this paper, it is assumed that an outage event occurs when the received SINR is less than a threshold SINR $\gamma_{\text {th }}[30,40]$. In addition, the data transmission in the secondary network is fixed to the delay-limited mode in which the secondary receivers such as the selected secondary relay $R_{m}$ and the secondary sources $S_{1}$ and $S_{2}$ decode the desired data block by block without using buffers [30, 40]. Throughput has been considered to characterize the spectrum utilization of the communication systems $[30,40,41]$ (known as the mean spectral efficiency) and is related to outage analyses as

$$
\begin{aligned}
\mathrm{TP}_{Z}^{(l)} & =\frac{1}{2}\left(1-\mathrm{OP}_{Z-S_{1}}^{(l)}\right) R_{\mathrm{th}}+\frac{1}{2}\left(1-\mathrm{OP}_{Z-S_{2}}^{(l)}\right) R_{\mathrm{th}} \\
& =\frac{1}{2}\left(2-\mathrm{OP}_{Z-S_{1}}^{(l)}-\mathrm{OP}_{Z-S_{2}}^{(l)}\right) R_{\mathrm{th}}
\end{aligned}
$$

where $1 / 2$ denotes that the $Z$ protocol operates in the two time slots, $Z \in\{\mathrm{UTW}-2 \mathrm{TS}-\mathrm{DNC}$, UTW-2TS-SC $\}$; $R_{\mathrm{th}}$ is the threshold data rate and is given by $R_{\mathrm{th}}=\log _{2}\left(1+\gamma_{\mathrm{th}}\right)$ (bits/s/Hz) [30, 38, 40, 41]; $\mathrm{OP}_{Z-S_{k}}^{(l)}$ is the outage probability at the secondary source $S_{k}$ in the case $l(l, k=\{1,2\})$ and is defined as probability that the secondary source $S_{k}$ cannot decode successfully the desired data. 


\subsection{The UTW-2TS-DNC Protocol}

3.1.1. Case 1: $d_{S_{1} R_{i}} \leq d_{S_{2} R_{i}}, i=\{1,2, \cdots, M\}$. The outage probability of the secondary source $S_{1}$ occurs when the secondary source $S_{1}$ cannot successfully decode the data $x_{2}$ of the opposite secondary source $S_{2}$. Due to the exploitation of the SIC technique at the selected secondary relay $R_{m}$ which was set up in the initial phase, the outage probability of the secondary source $S_{1}$ happens when (1) the $R_{m}$ fails to decode the data $x_{1}$; or (2) the $R_{m}$ decodes the data $x_{1}$ successfully but does not decode the data $x_{2}$; or (3) the $R_{m}$ decodes both $x_{1}$ and $x_{2}$ successfully, but the $S_{1}$ cannot decode the DNCcoded data $x$ to get the desired data $x_{2}$ in the second time slot. We express the outage probability of the secondary source $S_{1}$ by the mathematical expression

$$
\begin{aligned}
& \mathrm{OP}_{\mathrm{UTW}-2 \mathrm{TS}-\mathrm{DNC}-S_{1}}^{(1)}=\underbrace{\operatorname{Pr}\left\{\gamma_{R_{m} \rightarrow x_{1}}^{(1)}<\gamma_{\text {th }}\right\}}_{\Phi_{1}} \\
& +\underbrace{\operatorname{Pr}\left\{\left(\gamma_{R_{m} \rightarrow x_{1}}^{(1)} \geq \gamma_{\mathrm{th}}\right) \cap\left(\gamma_{R_{m} \rightarrow x_{2}}^{(1)}<\gamma_{\mathrm{th}}\right)\right\}}_{\Phi_{2}} \\
& +\operatorname{Pr}\left\{\left(\gamma_{R_{m} \rightarrow x_{1}}^{(1)} \geq \gamma_{\text {th }}\right) \cap\left(\gamma_{R_{m} \rightarrow x_{2}}^{(1)} \geq \gamma_{\text {th }}\right)\right. \\
& \left.\cdot \cap\left(\gamma_{S_{1} \rightarrow x}^{\mathrm{UTW}-2 \mathrm{TS}-\mathrm{DNC}}<\gamma_{\mathrm{th}}\right)\right\} \text {. }
\end{aligned}
$$

In (18), the probability $\Phi_{1}=\operatorname{Pr}\left\{\gamma_{R_{m} \rightarrow x_{1}}^{(1)}<\gamma_{\text {th }}\right\}$ is considered when the secondary relay $R_{m}$ fails to decode the data $x_{1}$ while applying the SIC technique. The probability $\Phi_{2}=\operatorname{Pr}$ $\left\{\left(\gamma_{R_{m} \rightarrow x_{1}}^{(1)} \geq \gamma_{\text {th }}\right) \cap\left(\gamma_{R_{m} \rightarrow x_{2}}^{(1)}<\gamma_{\text {th }}\right)\right\}$ is the decoding error of the data $x_{2}$ at the secondary relay $R_{m}$. Finally, the probability

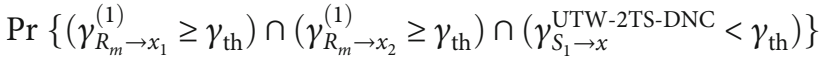
measures the decoding error of the coded data $x$ at the secondary source $S_{1}$. The event $\gamma_{S_{1} \rightarrow x}^{\text {SIC-DNC }}<\gamma_{\text {th }}$ occurs independently with the events $\gamma_{R_{m} \rightarrow x_{1}}^{(1)} \geq \gamma_{\text {th }}$ and $\gamma_{R_{m} \rightarrow x_{2}}^{(1)} \geq \gamma_{\text {th }}$; thus, we have an equivalent representation of (18) as

$$
\begin{aligned}
& \mathrm{OP}_{\text {UTW-2TS-DNC-S }}^{(1)}=\Phi_{1}+\Phi_{2}+\operatorname{Pr}\left\{\left(\gamma_{R_{m} \rightarrow x_{1}}^{(1)} \geq \gamma_{\text {th }}\right) \cap\left(\gamma_{R_{m} \rightarrow x_{2}}^{(1)} \geq \gamma_{\text {th }}\right)\right\} \\
& \times \operatorname{Pr}\left\{\gamma_{S_{1} \rightarrow x}^{\mathrm{UTW}-2 \mathrm{TS}-\mathrm{DNC}}<\gamma_{\text {th }}\right\} \\
& =\Phi_{1}+\Phi_{2}+\left(\operatorname{Pr}\left\{\gamma_{R_{m} \rightarrow x_{1}}^{(1)} \geq \gamma_{\text {th }}\right\}-\operatorname{Pr}\left\{\left(\gamma_{R_{m} \rightarrow x_{1}}^{(1)} \geq \gamma_{\text {th }}\right)\right.\right. \\
& \left.\left.\cdot \cap\left(\gamma_{R_{m} \rightarrow x_{2}}^{(1)}<\gamma_{\text {th }}\right)\right\}\right) \times \operatorname{Pr}\left\{\gamma_{S_{1} \rightarrow x}^{\text {UTW-2TS-DNC }}<\gamma_{\text {th }}\right\} \\
& =\Phi_{1}+\Phi_{2}+\left(1-\operatorname{Pr}\left\{\gamma_{R_{m} \rightarrow x_{1}}^{(1)}<\gamma_{\text {th }}\right\}\right. \\
& \left.-\operatorname{Pr}\left\{\left(\gamma_{R_{m} \rightarrow x_{1}}^{(1)} \geq \gamma_{\mathrm{th}}\right) \cap\left(\gamma_{R_{m} \rightarrow x_{2}}^{(1)}<\gamma_{\mathrm{th}}\right)\right\}\right) \\
& \times \operatorname{Pr}\left\{\gamma_{S_{1} \rightarrow x}^{\mathrm{UTW}-2 \mathrm{TS}-\mathrm{DNC}}<\gamma_{\text {th }}\right\} \\
& =\left(\Phi_{1}+\Phi_{2}\right)\left(1-\operatorname{Pr}\left\{\gamma_{S_{1} \rightarrow x}^{\text {UTW-2TS-DNC }}<\gamma_{\text {th }}\right\}\right) \\
& +\operatorname{Pr}\left\{\gamma_{S_{1} \rightarrow x}^{\mathrm{UTW}-2 \mathrm{TS}-\mathrm{DNC}}<\gamma_{\text {th }}\right\} \text {. }
\end{aligned}
$$

To analyze the probabilities $\Phi_{1}, \Phi_{2}$, and $\operatorname{Pr}\{$ $\left.\gamma_{S_{1} \rightarrow x}^{\text {UTW-2TS-DNC }}<\gamma_{\text {th }}\right\}$, we define $G_{X_{1} Y_{1} / X_{2} Y_{2}}=g_{X_{1} Y_{1}} / g_{X_{2} Y_{2}}$, where $X_{1}, X_{2}, Y_{1}, Y_{2} \in\left\{S_{1}, S_{2}, R_{i}, \mathrm{PR}\right\}, g_{X_{1} Y_{1}} \neq g_{X_{2} Y_{2}}$, and $i=\{1,2, \cdots, M\}$; then, by referring from [30] (eqs. 24-25), the CDF and PDF of the RV $G_{X_{1} Y_{1} / X_{2} Y_{2}}$ are obtained as

$$
\begin{aligned}
& F_{G_{X_{1} Y_{1} / X_{2} Y_{2}}}(z)=\frac{\lambda_{X_{1} Y_{1}} z}{\lambda_{X_{2} Y_{2}}+\lambda_{X_{1} Y_{1}} z}, \\
& f_{G_{X_{1} Y_{1} / X_{2} Y_{2}}}(x)=\frac{d F_{G_{X_{1} Y_{1} / X_{2} Y_{2}}}(x)}{d x}=\frac{\lambda_{X_{1} Y_{1}} \lambda_{X_{2} Y_{2}}}{\left(\lambda_{X_{2} Y_{2}}+\lambda_{X_{1} Y_{1}} x\right)^{2}} .
\end{aligned}
$$

For the selected secondary relay $R_{m}$ from the $S_{1}-R_{i}$ links $(m, i=\{1,2, \cdots, M\})$, the CDF of the RV $G_{S_{1} R_{m} / S_{1} \mathrm{PR}}$ is given as

$$
\begin{aligned}
F_{G_{S_{1} R_{m} / S_{1} \mathrm{PR}}}(z) & =\operatorname{Pr}\left\{G_{S_{1} R_{m} / S_{1} \mathrm{PR}}<z\right\}=\operatorname{Pr}\left\{\frac{g_{S_{1} R_{m}}}{g_{S_{1} \mathrm{PR}}}<z\right\} \\
& =\operatorname{Pr}\left\{g_{S_{1} R_{m}}<z g_{S_{1} \mathrm{PR}}\right\} \\
& =\int_{0}^{\infty} f_{g_{S_{1} \mathrm{PR}}}(t) \times F_{g_{S_{1} R_{m}}}(z t) d t .
\end{aligned}
$$

Substituting the CDF of the RV $g_{S_{1} R_{m}}$ in (4) and the PDF of the $\mathrm{RVg}_{S_{1} \mathrm{PR}}$ into (22), we have a final result as

$$
\begin{aligned}
F_{G_{S_{1} R_{m} / S_{1} \mathrm{PR}}}(z) & =\int_{0}^{\infty} \lambda_{4} e^{-\lambda_{4} t} \sum_{p=0}^{M}\left(\begin{array}{c}
p \\
M
\end{array}\right)(-1)^{p} e^{-p \lambda_{1} z t} d t \\
& =\lambda_{4} \sum_{p=0}^{M}\left(\begin{array}{c}
p \\
M
\end{array}\right)(-1)^{p} \int_{0}^{\infty} e^{-t\left(\lambda_{4}+p \lambda_{1} z\right)} d t \\
& =\lambda_{4} \sum_{p=0}^{M}\left(\begin{array}{c}
p \\
M
\end{array}\right) \frac{(-1)^{p}}{\lambda_{4}+p \lambda_{1} z} .
\end{aligned}
$$

The probabilities $\Phi_{1}$ and $\Phi_{2}$ are solved by Lemmas 1 and 2 as follows.

Lemma 1. The probability $\Phi_{1}$ is solved as

$$
\begin{aligned}
\Phi_{1}= & +\lambda_{2} \lambda_{4} \lambda_{5} \sum_{p=1}^{M}\left(\begin{array}{c}
p \\
M
\end{array}\right) \frac{(-1)^{p}}{\lambda_{2} v_{3}(p)-\lambda_{5} v_{4}(p)} \\
& \cdot\left(\frac{1}{\lambda_{5}}-\frac{v_{4}(p)}{\lambda_{2} v_{3}(p)-\lambda_{5} v_{4}(p)} \ln \left(\frac{\lambda_{2} v_{3}(p)}{\lambda_{5} v_{4}(p)}\right)\right),
\end{aligned}
$$

where $v_{1}=\gamma_{t h} \alpha_{2} / \alpha_{1}, v_{2}=\gamma_{t h} / \alpha_{1} Q, v_{3}(p)$, and $v_{4}(p)$ are functions versus count variable $p$ and are defined, respectively, as $v_{3}(p)=\lambda_{4}+p \lambda_{1} v_{2}$ and $v_{4}(p)=p \lambda_{1} v_{1}$.

Proof. (proven in Appendix A). 
Lemma 2. The probability $\Phi_{2}$ is given in two cases of $\varepsilon$ as follows:

Case $\varepsilon=0$ (perfect SICs):

$$
\begin{aligned}
\Phi_{2}= & \frac{\lambda_{2} v_{5}}{\lambda_{5}+\lambda_{2} v_{5}}-\lambda_{2} \lambda_{4} \lambda_{5} \sum_{p=0}^{M}\left(\begin{array}{c}
p \\
M
\end{array}\right) \frac{(-1)^{p}}{\lambda_{2} v_{3}(p)-\lambda_{5} v_{4}(p)} \\
& \times\left(\frac{\lambda_{2} v_{5}}{\lambda_{5}\left(\lambda_{5}+\lambda_{2} v_{5}\right)}-\frac{v_{4}(p)}{\lambda_{2} v_{3}(p)-\lambda_{5} v_{4}(p)} \ln \left(\frac{\left(\lambda_{5}+\lambda_{2} v_{5}\right) \times v_{3}(p)}{\lambda_{5} \times\left(v_{3}(p)+v_{5} v_{4}(p)\right)}\right)\right) .
\end{aligned}
$$

Cas $\varepsilon=1$ (imperfect SICs):

$$
\begin{aligned}
\Phi_{2}= & -\lambda_{5} e^{\left(\lambda_{5}+\lambda_{2} v_{5}\right) \Omega / \lambda_{2} v_{6}} \Gamma\left(0, \frac{\left(\lambda_{5}+\lambda_{2} v_{5}\right) \Omega}{\lambda_{2} v_{6}}\right)\left\{\frac{\Omega}{\lambda_{2} v_{6}}\right. \\
& \left.-\sum_{p=0}^{M}\left(\begin{array}{c}
p \\
M
\end{array}\right) \frac{(-1)^{p}}{\lambda_{2} v_{3}(p)-\lambda_{5} v_{4}(p)}\left(\frac{\Omega \lambda_{4}}{v_{6}}+\frac{\lambda_{2} \lambda_{4} v_{4}(p)}{\lambda_{2} v_{3}(p)-\lambda_{5} v_{4}(p)}\right)\right\} \\
& -\lambda_{2} \lambda_{4} \sum_{p=0}^{M}\left(\begin{array}{c}
p \\
M
\end{array}\right) \frac{(-1)^{p}}{\lambda_{2} v_{3}(p)-\lambda_{5} v_{4}(p)}\left\{1-\frac{\lambda_{5} v_{4}(p)}{\lambda_{2} v_{3}(p)-\lambda_{5} v_{4}(p)}\right. \\
& \times\left(\ln \left(\frac{\left(\lambda_{5}+\lambda_{2} v_{5}\right) v_{3}(p)}{\lambda_{5}\left(v_{3}(p)+v_{5} v_{4}(p)\right)}\right)\right. \\
& \left.\left.-e^{\left(v_{3}(p)+v_{5} v_{4}(p)\right) \Omega / v_{6} v_{4}(p)} \Gamma\left(0, \frac{\left(v_{3}(p)+v_{5} v_{4}(p)\right) \Omega}{v_{6} v_{4}(p)}\right)\right)\right\},
\end{aligned}
$$

where $\Gamma(u, v)$ is the upper incomplete Gamma function $\left(\Gamma(u, v)=\int_{v}^{\infty} e^{-t} t^{u-1} d t\right) \quad$ ([42], eq. 8.350.2).

Proof. (see the proof and notations in Appendix B).

The last probability $\operatorname{Pr}\left\{\gamma_{S_{1} \rightarrow x}^{\mathrm{UTW}-2 \mathrm{TS}-\mathrm{DNC}}<\gamma_{\mathrm{th}}\right\}$ in (19) is obtained by using formula (13) with $k=1$ as

$$
\begin{aligned}
\operatorname{Pr}\left\{\gamma_{S_{1} \rightarrow x}^{\mathrm{UTW}-2 \mathrm{TS}-\mathrm{DNC}}<\gamma_{\text {th }}\right\} & =\operatorname{Pr}\left\{\frac{Q g_{R_{m} S_{1}}}{g_{R_{m} \mathrm{PR}}}<\gamma_{\text {th }}\right\} \\
& =\operatorname{Pr}\left\{G_{R_{m} S_{1} / R_{m} \mathrm{PR}}<\frac{\gamma_{\mathrm{th}}}{Q}\right\} \\
& =F_{G_{R_{m} S_{1} / R_{m} \mathrm{PR}}}\left(\frac{\gamma_{\mathrm{th}}}{Q}\right) \\
& =\frac{\lambda_{1}\left(\gamma_{\mathrm{th}} / Q\right)}{\lambda_{3}+\lambda_{1}\left(\gamma_{\mathrm{th}} / Q\right)} \\
& =\frac{\lambda_{1} \gamma_{\mathrm{th}}}{\lambda_{3} Q+\lambda_{1} \gamma_{\mathrm{th}}} .
\end{aligned}
$$

Substituting (27) into (19), the outage probability of the secondary source $S_{1}$ is obtained by the closed-form expression

$$
\mathrm{OP}_{\mathrm{UTW}-2 \mathrm{TS}-\mathrm{DNC}-\mathrm{S}_{1}}^{(1)}=\frac{\left(\Phi_{1}+\Phi_{2}\right) \lambda_{3} Q+\lambda_{1} \gamma_{\mathrm{th}}}{\lambda_{3} Q+\lambda_{1} \gamma_{\mathrm{th}}},
$$

where $\Phi_{1}$ and $\Phi_{2}$ are given by closed-form expressions in Lemmas 1 and 2, respectively.
The secondary source $S_{2}$ expects to receive the desired data $x_{1}$ of the secondary source $S_{1}$. Hence, the outage probability of the secondary source $S_{2}$ occurs when (1) the selected secondary relay $R_{m}$ cannot decode the data $x_{1}$ or (2) the $S_{2}$ fails to decode the data transmitted by the $R_{m}$ after the data $x_{1}$ is decoded successfully. We formulate the outage probability of the secondary source $S_{1}$ as

$$
\begin{aligned}
\mathrm{OP}_{\mathrm{UTW}-2 T S-D N C-S_{2}}^{(1)}= & \operatorname{Pr}\left\{\gamma_{R_{m} \rightarrow x_{1}}^{(1)}<\gamma_{\text {th }}\right\}+\operatorname{Pr}\left\{\left(\gamma_{R_{m} \rightarrow x_{1}}^{(1)} \geq \gamma_{\text {th }}\right)\right. \\
& \left.\cdot \cap\left(\gamma_{S_{2} \rightarrow x}^{\mathrm{UTW}-2 \mathrm{TS}-\mathrm{DNC}}<\gamma_{\text {th }}\right)\right\} .
\end{aligned}
$$

In (29), the probability part $\operatorname{Pr}\left\{\left(\gamma_{R_{m} \rightarrow x_{1}}^{(1)} \geq \gamma_{\text {th }}\right) \cap(\right.$ $\left.\left.\gamma_{S_{2} \rightarrow x}^{\text {UTW-2TS-DNC }}<\gamma_{\text {th }}\right)\right\}$ shows transmission errors of the data $x_{1}$ from the selected secondary relay $R_{m}$ to the desired secondary source $S_{2}$. Because the event $\gamma_{S_{2} \rightarrow x}^{\mathrm{UTW}-2 \mathrm{TS}-\mathrm{DNC}}<\gamma_{\text {th }}$ occurs independently, and with the help of (13) with $k=1$, the outage probability of the secondary source $S_{2}$ is manipulated and solved as

$$
\begin{aligned}
\mathrm{OP}_{\mathrm{UTW}-2 \mathrm{TS}-\mathrm{DNC}-S_{2}}^{(1)}= & \Phi_{1}+\operatorname{Pr}\left\{\gamma_{R_{m} \rightarrow x_{1}}^{(1)} \geq \gamma_{\mathrm{th}}\right\} \\
& \times \operatorname{Pr}\left\{\gamma_{S_{2} \rightarrow x}^{\mathrm{UTW}-2 \mathrm{TS}-\mathrm{DNC}}<\gamma_{\mathrm{th}}\right\} \\
= & \Phi_{1}+\left(1-\Phi_{1}\right) \times \operatorname{Pr}\left\{\frac{Q g_{R_{m} S_{2}}}{g_{R_{m} \mathrm{PR}}}<\gamma_{\mathrm{th}}\right\} \\
= & \Phi_{1}+\left(1-\Phi_{1}\right) \times F_{G_{R_{m} S_{2} / R_{m} \mathrm{PR}}}\left(\frac{\gamma_{\mathrm{th}}}{Q}\right) \\
= & \Phi_{1}+\left(1-\Phi_{1}\right) \times\left(\frac{\lambda_{2} \gamma_{\mathrm{th}}}{\lambda_{3} Q+\lambda_{2} \gamma_{\mathrm{th}}}\right),
\end{aligned}
$$

where $\Phi_{1}$ is given exactly by Lemma 1 .

Next, the throughput of the UTW-2TS-DNC protocol as in (17) is solved in Case $1\left(d_{S_{1} R_{i}} \leq d_{S_{2} R_{i}}\right)$ by

$$
\begin{aligned}
\mathrm{TP}_{\mathrm{UTW}-2 T S-D N C}^{(1)}= & \frac{1}{2}\left(2-\mathrm{OP}_{\mathrm{UTW}-2 T S-D N C-S_{1}}^{(1)}-\mathrm{OP}_{\mathrm{UTW}-2 \text { TS-DNC-S }}^{(1)}\right) \\
& \cdot \log _{2}\left(1+\gamma_{\mathrm{th}}\right) \\
= & \frac{\log _{2}\left(1+\gamma_{\mathrm{th}}\right)}{2}\left\{2-2 \Phi_{1}-\left(1-\Phi_{1}\right)\right. \\
& \cdot\left(\frac{\lambda_{1} \gamma_{\mathrm{th}}}{\lambda_{3} Q+\lambda_{1} \gamma_{\mathrm{th}}}+\frac{\lambda_{2} \gamma_{\mathrm{th}}}{\lambda_{3} Q+\lambda_{2} \gamma_{\mathrm{th}}}\right) \\
& \left.-\frac{\lambda_{3} Q \Phi_{2}}{\lambda_{3} Q+\lambda_{1} \gamma_{\mathrm{th}}}\right\}
\end{aligned}
$$

where $\Phi_{1}$ and $\Phi_{2}$ are taken from Lemmas 1 and 2, respectively.

3.1.2. Case 2: $d_{S_{1} R_{i}}>d_{S_{2} R_{i}}$. In this case, a selected secondary relay $R_{m}$ must decode the data $x_{2}$ firstly. Because the system model of the UTW-2TS-DNC protocol in Figure 1 is 
symmetric, we can model the outage probabilities in Case 1 as functions versus symmetric parameters as $\mathrm{OP}_{\mathrm{UTW}-2 \text { TS-DNC-S }}^{(1)} \equiv \mathrm{OP}_{\mathrm{UTW}-2 \text { TS-DNC-S }}^{(1)}\left(\lambda_{1}, \alpha_{1}, \lambda_{2}, \alpha_{2}\right)$ and $\mathrm{OP}_{\mathrm{UTW}-2 \mathrm{TS}-\mathrm{DNC}-S_{2}}^{(1)} \equiv \mathrm{OP}_{\mathrm{UTW}-2 \mathrm{TS} \text {-DNC- } \mathrm{S}_{2}}^{(1)}\left(\lambda_{1}, \alpha_{1}, \lambda_{2}, \alpha_{2}\right)$. Hence, in Case $2\left(d_{S_{1} R_{i}}>d_{S_{2} R_{i}}\right)$, the outage probability of the secondary sources $S_{1}$ and $S_{2}$ can be inferred from Case 1 as follows:

$$
\begin{aligned}
& \mathrm{OP}_{\mathrm{UTW}-2 \text { TS-DNC-S }}^{(2)}=\mathrm{OP}_{\mathrm{UTW}-2 \text { TS-DNC-S }}^{(1)}\left(\lambda_{2}, \alpha_{2}, \lambda_{1}, \alpha_{1}\right), \\
& \mathrm{OP}_{\mathrm{UTW}-2 \mathrm{TS}-\mathrm{DNC}-\mathrm{S}_{2}}^{(2)}=\mathrm{OP}_{\mathrm{UTW}-2 \text { TS-DNC-S }}^{(1)}\left(\lambda_{2}, \alpha_{2}, \lambda_{1}, \alpha_{1}\right),
\end{aligned}
$$

where $\mathrm{OP}_{\mathrm{UTW}-2 \mathrm{TS}-\mathrm{DNC} \mathrm{S}_{2}}^{(1)}\left(\lambda_{2}, \alpha_{2}, \lambda_{1}, \alpha_{1}\right)$ and $\mathrm{OP}_{\mathrm{UTW}-2 \mathrm{TS}-\mathrm{DNC}-\mathrm{S}_{1}}^{(1)}$ $\left(\lambda_{2}, \alpha_{2}, \lambda_{1}, \alpha_{1}\right)$ are closed-form outage probability expressions of the secondary sources $S_{2}$ and $S_{1}$ in Case 1 with replacing $\lambda_{1} \leftrightarrow \lambda_{2}$ and $\alpha_{1} \leftrightarrow \alpha_{2}$.

The throughput of the UTW-2TS-DNC protocol as in (17) is obtained in Case 2 by

$$
\begin{aligned}
\mathrm{TP}_{\mathrm{UTW}-2 \mathrm{TS}-\mathrm{DNC}}^{(2)}= & \frac{\log _{2}\left(1+\gamma_{\mathrm{th}}\right)}{2} \\
& \cdot\left(2-\mathrm{OP}_{\mathrm{UTW}-2 \mathrm{TS}-\text { DNC-S }}^{(2)}-\mathrm{OP}_{\mathrm{UTW}-2 \mathrm{TS}-\mathrm{DNC}-\mathrm{S}_{2}}^{(2)}\right),
\end{aligned}
$$

where $\mathrm{OP}_{\mathrm{UTW}-2 \mathrm{TS}-\mathrm{DNC}-\mathrm{S}_{1}}^{(2)}$ and $\mathrm{OP}_{\mathrm{UTW}-2 \mathrm{TS}-\mathrm{DNC}-\mathrm{S}_{2}}^{(2)}$ are presented in (32) and (33), respectively.

3.2. The UTW-2TS-SC Protocol. Operation of the UTW-2TS$\mathrm{SC}$ protocol is different to the UTW-2TS-DNC protocol in the time slot 2 (the last time slot) with power allocation coefficients $\beta_{1}$ and $\beta_{2}$ to decoded data $x_{1}$ and $x_{2}$. Similar to the UTW-2TS-DNC protocol for Case $1\left(d_{S_{1} R_{i}} \leq d_{S_{2} R_{i}}\right)$ and Case $2\left(d_{S_{1} R_{i}}>d_{S_{2} R_{i}}\right)$, the outage probability and corresponding throughput of the secondary sources $S_{1}$ and $S_{2}$ will be obtained briefly in the next subsections.

3.2.1. Case 1: $d_{S_{1} R_{i}} \leq d_{S_{2} R_{i}}$. The decoding operation of the UTW-2TS-SC protocol is similar to that of the UTW-2TS$\mathrm{DNC}$ protocol. One difference is that the secondary source $S_{1}$ expects to decode the desired data $x_{2}$ directly from the received signal $y_{S_{1}}$ as in (14) where $k=1$ and $l=2$. Hence, the outage probability of the secondary source $S_{1}$ (denoted as $\left.\mathrm{OP}_{\mathrm{UTW}-2 \mathrm{TS}-\mathrm{SC}-S_{1}}^{(1)}\right)$ is expressed in Case $1\left(d_{S_{1} R_{i}} \leq d_{S_{2} R_{i}}\right)$ as

$$
\begin{aligned}
& \mathrm{OP}_{\mathrm{UTW}-2 \mathrm{TS}-\mathrm{SC}-\mathrm{S}_{1}}^{(1)}=\operatorname{Pr}\left\{\gamma_{R_{m} \rightarrow x_{1}}^{(1)}<\gamma_{\text {th }}\right\} \\
& +\operatorname{Pr}\left\{\left(\gamma_{R_{m} \rightarrow x_{1}}^{(1)} \geq \gamma_{\text {th }}\right) \cap\left(\gamma_{R_{m} \rightarrow x_{2}}^{(1)}<\gamma_{\text {th }}\right)\right\} \\
& +\operatorname{Pr}\left\{\left(\gamma_{R_{m} \rightarrow x_{1}}^{(1)} \geq \gamma_{\text {th }}\right) \cap\left(\gamma_{R_{m} \rightarrow x_{2}}^{(1)} \geq \gamma_{\text {th }}\right)\right. \\
& \left.\cdot \cap\left(\gamma_{S_{1} \rightarrow x_{2}}^{\mathrm{UTW}-2 \mathrm{TS}-\mathrm{SC}}<\gamma_{\text {th }}\right)\right\} \\
& =\left(\Phi_{1}+\Phi_{2}\right) \times\left(1-\operatorname{Pr}\left\{\gamma_{S_{1} \rightarrow x_{2}}^{\mathrm{UTW}-2 \mathrm{TS}-\mathrm{SC}}<\gamma_{\mathrm{th}}\right\}\right) \\
& +\operatorname{Pr}\left\{\gamma_{S_{1} \rightarrow x_{2}}^{\mathrm{UTW}-2 \mathrm{TS}-\mathrm{SC}}<\gamma_{\text {th }}\right\} \text {, }
\end{aligned}
$$

where $\Phi_{1}$ and $\Phi_{2}$ are obtained from Lemmas 1 and 2 .
In (35), the probability $\operatorname{Pr}\left\{\left(\gamma_{R_{m} \rightarrow x_{1}}^{(1)} \geq \gamma_{\text {th }}\right) \cap\left(\gamma_{R_{m} \rightarrow x_{2}}^{(1)} \geq\right.\right.$ $\left.\left.\gamma_{\mathrm{th}}\right) \cap\left(\gamma_{S_{1} \rightarrow x_{2}}^{\mathrm{UTW}-2 \mathrm{TS}-\mathrm{SC}}<\gamma_{\mathrm{th}}\right)\right\}$ directly calculates the decoding error of the data $x_{2}$ at the secondary source $S_{1}$.

Substituting (16) with $k=1$ and $l=2$ into the probability $\operatorname{Pr}\left\{\gamma_{S_{1} \rightarrow x_{2}}^{\mathrm{UTW}-2 T S-S C}<\gamma_{\text {th }}\right\}$ in (35) and performing similarly as (27), we have a result as

$$
\begin{aligned}
\operatorname{Pr}\left\{\gamma_{S_{1} \rightarrow x_{2}}^{\mathrm{UTW}-2 \mathrm{TS}-\mathrm{SC}}<\gamma_{\mathrm{th}}\right\} & =\operatorname{Pr}\left\{\frac{Q \beta_{2} g_{R_{m} S_{1}}}{g_{R_{m} \mathrm{PR}}}<\gamma_{\mathrm{th}}\right\} \\
& =\operatorname{Pr}\left\{G_{R_{m} S_{1} / R_{m} \mathrm{PR}}<\frac{\gamma_{\mathrm{th}}}{\beta_{2} Q}\right\} \\
& =F_{G_{R_{m}} S_{1} / R_{m} \mathrm{PR}}\left(\frac{\gamma_{\mathrm{th}}}{\beta_{2} Q}\right) \\
& =\frac{\lambda_{1}\left(\gamma_{\mathrm{th}} /\left(\beta_{2} Q\right)\right)}{\lambda_{3}+\lambda_{1}\left(\gamma_{\mathrm{th}} /\left(\beta_{2} Q\right)\right)} \\
& =\frac{\lambda_{1} \gamma_{\mathrm{th}}}{\lambda_{3} \beta_{2} Q+\lambda_{1} \gamma_{\mathrm{th}}} .
\end{aligned}
$$

Hence, the outage probability $\mathrm{OP}_{\mathrm{UTW}-2 \mathrm{TS}-\mathrm{SC}-S_{1}}^{(1)}$ is solved in a closed-form expression with $\Phi_{1}, \Phi_{2}$, and $\operatorname{Pr}\left\{\gamma_{S_{1} \rightarrow x_{2}}^{\text {UTW-2TS-SC }}\right.$ $\left.<\gamma_{\text {th }}\right\}$ from Lemmas 1 and 2 and (36).

We see that because $\beta_{2} \leq 1$, then $\operatorname{Pr}\left\{\gamma_{S_{1} \rightarrow x_{2}}^{\mathrm{UTW}-2 \mathrm{TS}-\mathrm{SC}}<\gamma_{\mathrm{th}}\right\}$ $\geq \operatorname{Pr}\left\{\gamma_{S_{1} \rightarrow x}^{\text {UTW-2TS-DNC }}<\gamma_{\text {th }}\right\}$ which leads to OP UTW-2TS-SC-S $_{1} \geq$ $\mathrm{OP}_{\mathrm{UTW}-2 \mathrm{TS}-\mathrm{DNC}-\mathrm{S}_{1} \text {. }}^{(1)}$

Similarly, the remaining outage probability of the secondary source $S_{2}$ is obtained as

$$
\begin{aligned}
\mathrm{OP}_{\mathrm{UTW}-2 \mathrm{TS}-S C-S_{2}}^{(1)}= & \operatorname{Pr}\left\{\gamma_{R_{m} \rightarrow x_{1}}^{(1)}<\gamma_{\mathrm{th}}\right\}+\operatorname{Pr}\left\{\left(\gamma_{R_{m} \rightarrow x_{1}}^{(1)} \geq \gamma_{\mathrm{th}}\right)\right. \\
& \left.\cdot \cap\left(\gamma_{S_{2} \rightarrow x_{1}}^{\mathrm{UTW}-2 \mathrm{TS}-\mathrm{SC}}<\gamma_{\mathrm{th}}\right)\right\} \\
= & \Phi_{1}+\left(1-\Phi_{1}\right) \times\left(\frac{\lambda_{2} \gamma_{\mathrm{th}}}{\lambda_{3} \beta_{1} Q+\lambda_{2} \gamma_{\mathrm{th}}}\right) .
\end{aligned}
$$

Then, the throughput of the UTW-2TS-SC protocol is solved in Case $1\left(d_{S_{1} R_{i}} \leq d_{S_{2} R_{i}}\right)$ by

$$
\begin{aligned}
\mathrm{TP}_{\mathrm{UTW}-2 \mathrm{TS}-\mathrm{SC}}^{(1)}= & \frac{1}{2}\left(2-\mathrm{OP}_{\mathrm{UTW}-2 \text { TS-SC-S }}^{(1)}-\mathrm{OP}_{\mathrm{UTW}-2 \mathrm{TS}-S C-S_{2}}^{(1)}\right) \\
& \cdot \log _{2}\left(1+\gamma_{\mathrm{th}}\right) \\
= & \frac{\log _{2}\left(1+\gamma_{\mathrm{th}}\right)}{2}\left\{2-2 \Phi_{1}-\left(1-\Phi_{1}\right)\right. \\
& \cdot\left(\frac{\lambda_{1} \gamma_{\mathrm{th}}}{\lambda_{3} \beta_{2} Q+\lambda_{1} \gamma_{\mathrm{th}}}+\frac{\lambda_{2} \gamma_{\mathrm{th}}}{\lambda_{3} \beta_{2} Q+\lambda_{2} \gamma_{\mathrm{th}}}\right) \\
& \left.-\frac{\lambda_{3} \beta_{2} Q \Phi_{2}}{\lambda_{3} \beta_{2} Q+\lambda_{1} \gamma_{\mathrm{th}}}\right\} .
\end{aligned}
$$

3.2.2. Case 2: $d_{S_{1} R_{i}}>d_{S_{2} R_{i}}$. We can also model the outage probabilities in Case 1 as functions versus parameters as 


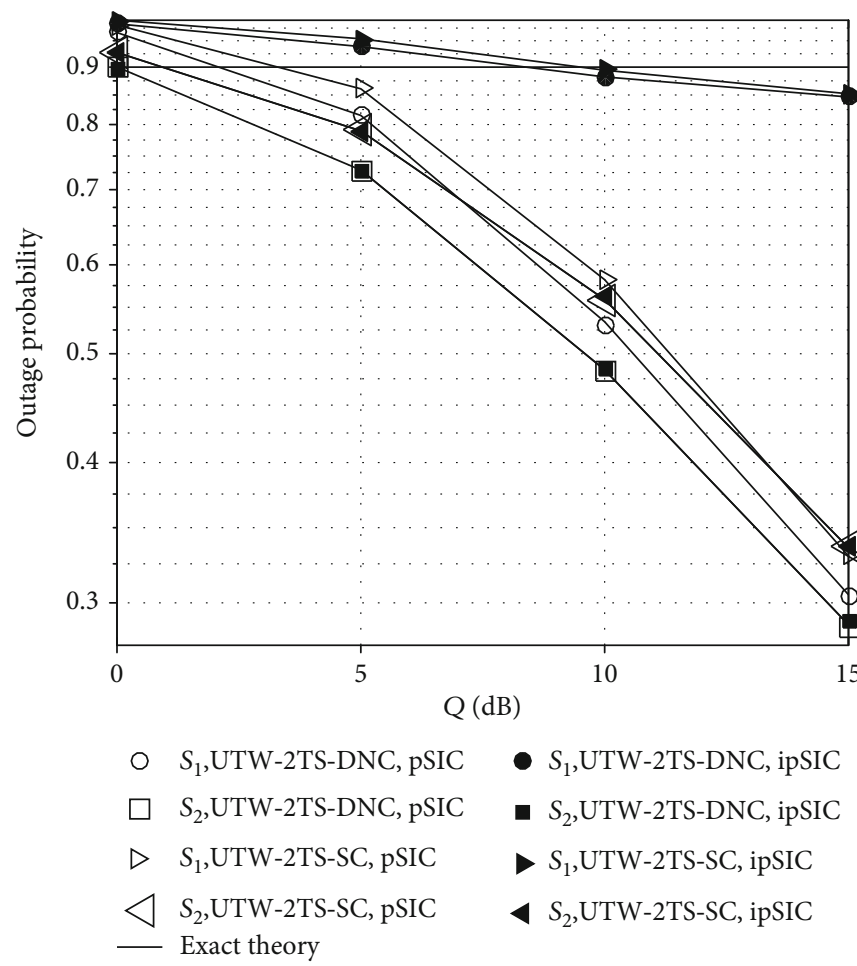

FIgURE 2: Outage probabilities of the secondary sources $S_{1}$ and $S_{2}$ versus $Q(\mathrm{~dB})$ when $\alpha_{1}=\alpha_{2}=0.5, \beta_{1}=0.7, \beta_{2}=1-\beta_{1}=0.3, \Psi=0$ (dB), $x_{R}=0.3, y_{R}=0, x_{\mathrm{PR}}=y_{\mathrm{PR}}=0.5$, and $M=3$.

$\mathrm{OP}_{\mathrm{UTW}-2 \mathrm{TS}-\mathrm{SC}-\mathrm{S}_{1}}^{(1)}\left(\lambda_{1}, \alpha_{1}, \beta_{1}, \lambda_{2}, \alpha_{2}, \beta_{2}\right)$ and $\mathrm{OP}_{\mathrm{UTW}-2 \mathrm{TS}-\mathrm{SC}-\mathrm{S}_{2}}^{(1)}($ $\left.\lambda_{1}, \alpha_{1}, \beta_{1}, \lambda_{2}, \alpha_{2}, \beta_{2}\right)$. Because of the symmetry model of the UTW-2TS-SC protocol in Figure 1, the outage probability of the secondary sources $S_{1}$ and $S_{2}$ in Case 2 can be concluded from Case 1 as follows:

$$
\mathrm{OP}_{\mathrm{UTW}-2 \mathrm{TS}-\mathrm{SC}-\mathrm{S}_{1}}^{(2)}=\mathrm{OP}_{\mathrm{UTW}-2 \mathrm{TS}-\mathrm{SC}-\mathrm{S}_{2}}^{(1)}\left(\lambda_{2}, \alpha_{2}, \beta_{2}, \lambda_{1}, \alpha_{1}, \beta_{1}\right) \text {, }
$$

$$
\mathrm{OP}_{\mathrm{UTW}-2 \mathrm{TS}-\mathrm{SC}-\mathrm{S}_{2}}^{(2)}=\mathrm{OP}_{\mathrm{UTW}-2 \mathrm{TS}-\mathrm{SC}-\mathrm{S}_{1}}^{(1)}\left(\lambda_{2}, \alpha_{2}, \beta_{2}, \lambda_{1}, \alpha_{1}, \beta_{1}\right) \text {, }
$$

where $\quad \operatorname{OP}_{\mathrm{UTW}-2 \text { TS-SC-S }}^{(1)}\left(\lambda_{2}, \alpha_{2}, \beta_{2}, \lambda_{1}, \alpha_{1}, \beta_{1}\right) \quad$ and $\mathrm{OP}_{\text {UTW-2TS-SC-S }}^{(1)}\left(\lambda_{2}, \alpha_{2}, \beta_{2}, \lambda_{1}, \alpha_{1}, \beta_{1}\right)$ are closed-form outage probability expressions of the secondary sources $S_{2}$ and $S_{1}$ in Case 1 with replacing $\lambda_{1} \leftrightarrow \lambda_{2}, \alpha_{1} \leftrightarrow \alpha_{2}$, and $\beta_{1} \leftrightarrow \beta_{2}$.

The corresponding throughput of the UTW-2TS-SC protocol is obtained in Case 2 as

$$
\begin{aligned}
\mathrm{TP}_{\mathrm{UTW}-2 \mathrm{TS}-\mathrm{SC}}^{(2)}= & \frac{\log _{2}\left(1+\gamma_{\mathrm{th}}\right)}{2} \\
& \cdot\left(2-\mathrm{OP}_{\mathrm{UTW}-2 \mathrm{TS}-S C-S_{1}}^{(2)}-\mathrm{OP}_{\mathrm{UTW}-2 \mathrm{TS}-\mathrm{SC}_{\mathrm{S}} \mathrm{S}_{2}}^{(2)}\right)
\end{aligned}
$$

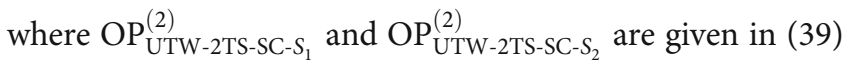
and (40), respectively.

\section{Results and Discussions}

This section presents analysis and simulation results in terms of outage probabilities and throughputs of the protocols UTW-2TS-DNC and UTW-2TS-SC in the two-dimensional plane. These proposed protocols are considered in two cases of perfect SICs $(\varepsilon=0$, denoted by pSIC) and imperfect SICs $(\varepsilon=1$, denoted by ipSIC). The simulation results are performed by the Monte Carlo method to validate the analysis ones which are shown from exact closed-form expressions. Coordinates of the nodes $S_{1}, S_{2}, \mathrm{PR}$, and the cluster with $M$ secondary relay $R_{i}$ are set as $S_{1}(0,0), S_{2}(1,0), \operatorname{PR}\left(x_{\mathrm{PR}}, y_{\mathrm{PR}}\right)$, and $R_{i}\left(x_{R}, y_{R}\right)$, where $0<x_{R}<1$ and $i=\{1,2, \cdots, M\}$. The normalized distances are calculated from the coordinates as $\quad d_{1}=\sqrt{x_{R}^{2}+y_{R}^{2}}, \quad d_{2}=\sqrt{\left(1-x_{R}\right)^{2}+y_{R}^{2}}, \quad d_{3}=$ $\sqrt{\left(x_{\mathrm{PR}}-x_{R}\right)^{2}+\left(y_{\mathrm{PR}}-y_{R}\right)^{2}}, \quad d_{4}=\sqrt{x_{\mathrm{PR}}^{2}+y_{\mathrm{PR}}^{2}}, \quad$ and $d_{5}=$ $\sqrt{\left(1-x_{\mathrm{PR}}\right)^{2}+y_{\mathrm{PR}}^{2}}$. It is assumed that the threshold SINR and the path loss exponent are fixed by $\gamma_{\text {th }}=3$ and $\eta=3$, and $Q(\mathrm{~dB})$ on the $x$-axis is defined as $Q=10 \times \log _{10}\left(I / \sigma^{2}\right)$ $(\mathrm{dB})$. Markers denote simulated results, and solid lines present analyzed ones.

Figure 2 shows the outage probabilities of the secondary sources $S_{1}$ and $S_{2}$ in the protocols UTW-2TS-DNC and UTW-2TS-SC versus $Q(\mathrm{~dB})$ when $x_{R}=0.3, y_{R}=0, x_{\mathrm{PR}}=$ $y_{\mathrm{PR}}=0.5, \Psi=0(\mathrm{~dB}), \quad M=3$, and interference allocation coefficients $\alpha_{1}=\alpha_{2}=0.5[19,30]$. The normalized distances are calculated as $d_{1}=0.3, d_{2}=0.7, d_{3}=0.54, d_{4}=0.71$, and $d_{5}=0.71$; hence, the results are presented following Case 1 

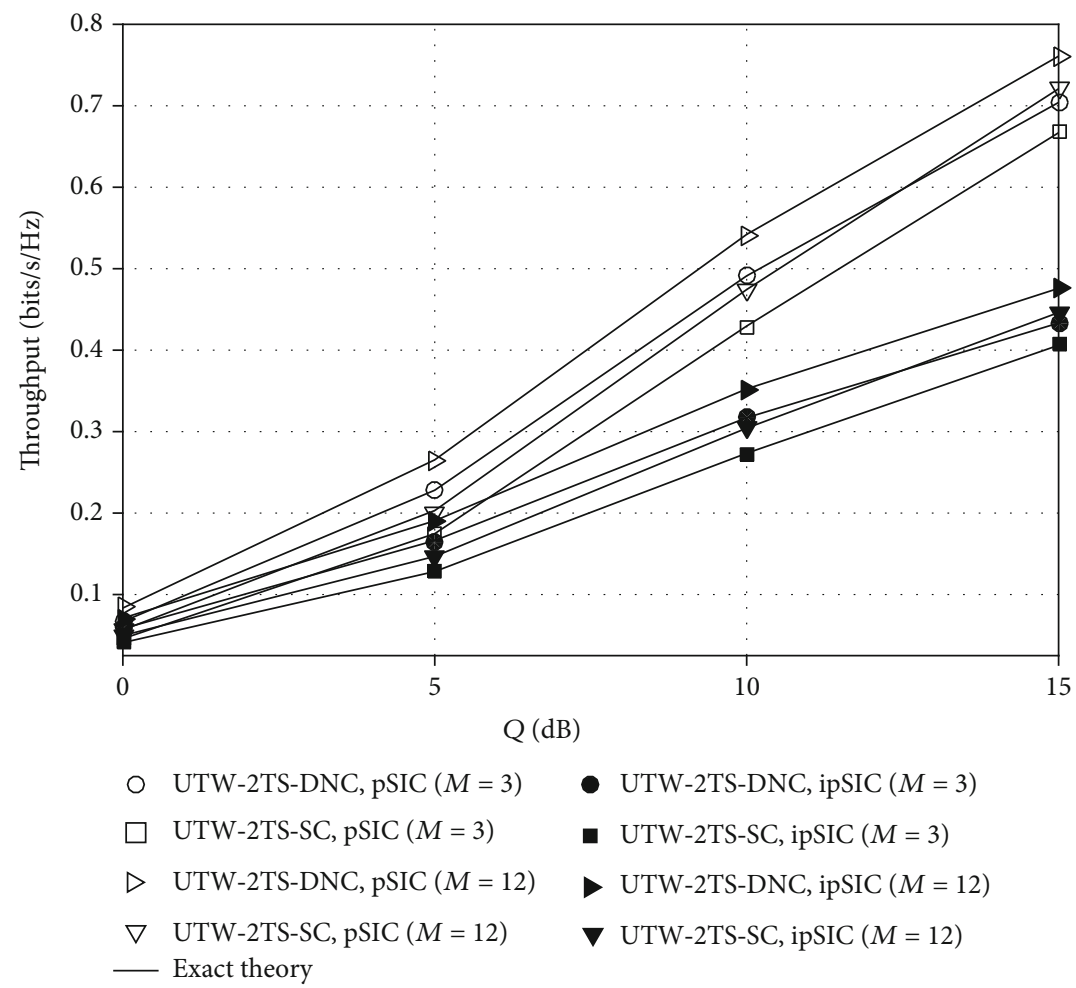

Figure 3: Throughputs of the protocols UTW-2TS-DNC and UTW-2TS-SC versus $Q$ (dB) when $\alpha_{1}=\alpha_{2}=0.5, \beta_{1}=0.7, \beta_{2}=1-\beta_{1}=0.3$, $\Psi=0(\mathrm{~dB}), x_{R}=0.3, y_{R}=0, x_{\mathrm{PR}}=y_{\mathrm{PR}}=0.5$, and $M=\{3,12\}$.

$\left(d_{S_{1} R_{i}} \leq d_{S_{2} R_{i}}\right)$. The power allocation coefficients $\beta_{1}$ and $\beta_{2}$ at the selected secondary relay are suitably set as $\beta_{1}=0.7$ and $\beta_{2}=1-\beta_{1}=0.3$. We note that the power allocation coefficients $\beta_{1}$ and $\beta_{2}$ to carry the desired data $x_{1}$ and $x_{2}$ from the selected relay to the $S_{2}$ and $S_{1}$, respectively. Simulated results are performed from equations (18) and (29) by making RVs in (3), (7), and (13), whereas exact theory values are calculated by closed-form expressions in (28) and (30) for the secondary sources $S_{1}$ and $S_{2}$, respectively. From Figure 2, some findings are watched as follows. Firstly, the outage probabilities of the secondary source $S_{1}$ and $S_{2}$ in both protocols with the perfect SICs and imperfect SICs decrease when the interference constraint parameters $Q$ increase. Secondly, the system performances of the secondary source $S_{1}$ with the perfect SICs are always better than those with the imperfect SICs. We note that the outage probabilities of the secondary source $S_{2}$ in both protocols are not affected by SIC operations, and thus, the system performances of the secondary source $S_{2}$ with the perfect SICs and the imperfect SICs are identical (markers and solid line are merged in Figure 2). Thirdly, in this case, the outage probabilities of the secondary source $S_{2}$ are almost smaller than those of the secondary source $S_{1}$. Finally, the results (solid lines) from the theory expressions of the outage probabilities are exact to the Monte Carlo simulations. These findings can be reasoned that the received SINRs in (3) and (7) to decode the data $x_{1}$ and $x_{2}$ declined by more interferences from transmittance of the data-carried signal $x_{2}$, affecting of the residual interference signal and interference constraints as in (1). In addition, with large values of $Q$, the transmit powers of the nodes $S_{1}, S_{2}$, and $R_{m}$ increase as in (1) which correspond to large SINRs to decode the data $x_{1}, x_{2}$, and $x$ as in (3), (7), (13), and (16), where $m=\{1,2, \cdots, M\}$.

Figure 3 compares the throughputs of the UTW-2TSDNC and UTW-2TS-SC protocols versus $Q(\mathrm{~dB})$ when $x_{R}$ $=0.3, y_{R}=0, x_{\mathrm{PR}}=y_{\mathrm{PR}}=0.5, \Psi=0(\mathrm{~dB}), \alpha_{1}=\alpha_{2}=0.5, \beta_{1}$ $=0.7, \beta_{2}=1-\beta_{1}=0.3$, and the number of intermediate secondary relays is set as $M \in\{3,12\}$. Exact throughput curves of the UTW-2TS-DNC and UTW-2TS-SC protocols are obtained by theoretic analyses in (31) and (38). Simulation markers are carried out by (17) with separate outage probabilities for each protocol. From Figure 3, the throughputs of the UTW-2TS-DNC protocol are larger than those of the UTW-2TS-SC protocol with all the cases of SICs and M. In addition, the throughputs of both protocols increase when the UTW-2TS systems use more secondary relays ( $M$ is increased). These contributions are based on observations from SINRs (13) and (16). With $0 \leq \beta_{1}$ and $\beta_{2} \leq 1$, the SINRs in (13) of the UTW-2TW-DNC protocol are always larger than those in (16) of the UTW-2TW-SC protocol. Furthermore, with more cooperative secondary relays, the diversity capacity of both protocols will be increased by the relay selection methods.

Figure 4 presents the throughputs of the protocols UTW2TS-DNC and UTW-2TS-SC versus $\alpha_{1}$ when $Q=10(\mathrm{~dB})$, $\beta_{1}=0.7, \beta_{2}=1-\beta_{1}=0.3, \Psi=-5(\mathrm{~dB}), x_{R}=0.3, y_{R}=0, x_{\mathrm{PR}}$ $=y_{\mathrm{PR}}=0.5, M=3$, and $\alpha_{2}$ can be set as $\alpha_{2}=1-\alpha_{1}$. In Figure 4 , the throughputs of the protocols UTW-2TS-DNC 


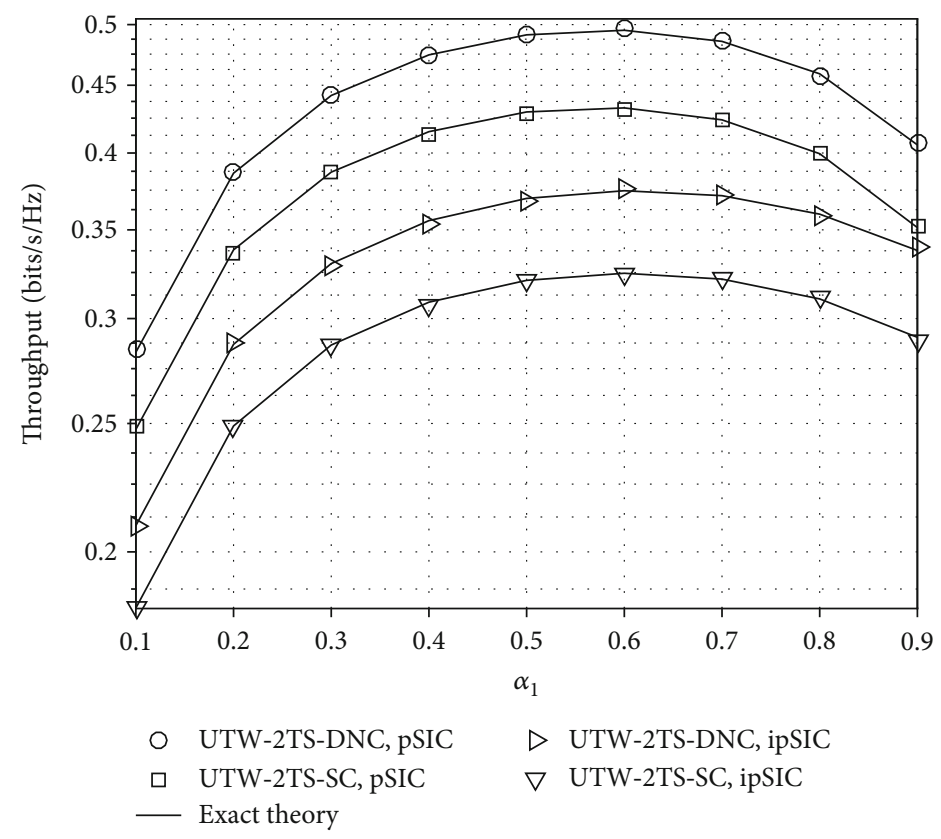

Figure 4: Throughputs of the protocols UTW-2TS-DNC and UTW-2TS-SC versus $\alpha_{1}$ when $Q=10(\mathrm{~dB}), \beta_{1}=0.7, \beta_{2}=1-\beta_{1}=0.3, \Psi=-5$ $(\mathrm{dB}), x_{R}=0.3, y_{R}=0, x_{\mathrm{PR}}=y_{\mathrm{PR}}=0.5, M=3$, and $\alpha_{2}=1-\alpha_{1}$.

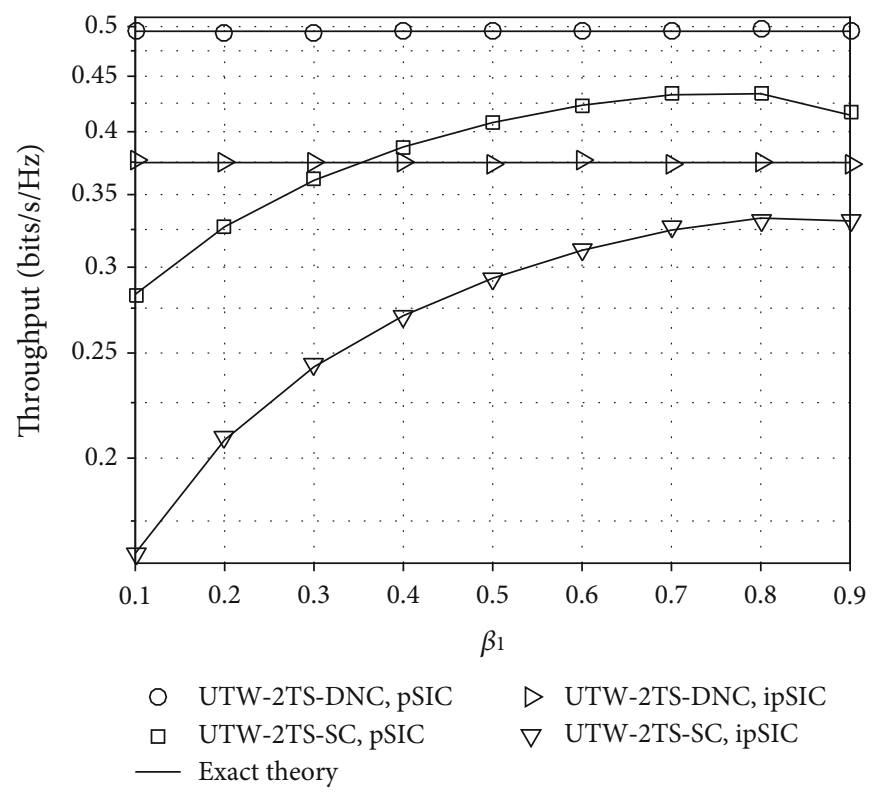

Figure 5: Throughputs of the protocols UTW-2TS-DNC and UTW-2TS-SC versus $\beta_{1}$ when $Q=10(\mathrm{~dB}), \alpha_{1}=0.6, \alpha_{2}=1-\alpha_{1}=0.4, \Psi=-5$ $(\mathrm{dB}), x_{R}=0.3, y_{R}=0, x_{\mathrm{PR}}=y_{\mathrm{PR}}=0.5, M=3$, and $\beta_{2}=1-\beta_{1}$.

and UTW-2TS-SC achieve the large values at an approximate interference power allocation coefficient $\alpha_{1} \approx 0.6$ for both cases (perfect SICs and imperfect SICs). The value $\alpha_{1} \approx 0.6$ is to balance constraints such as interferences from the secondary network to the primary network, locations of the secondary relays, and perfect/imperfect SIC operations.

Figure 5 shows the throughputs of the protocols UTW-2TS-DNC and UTW-2TS-SC versus $\beta_{1}$ when $Q=$ $10(\mathrm{~dB}), \alpha_{1}=0.6, \alpha_{2}=1-\alpha_{1}=0.4, \Psi=-5(\mathrm{~dB}), x_{R}=0.3$, $y_{R}=0, x_{\mathrm{PR}}=y_{\mathrm{PR}}=0.5, M=3$, and $\beta_{2}$ is changed as $\beta_{2}=$ $1-\beta_{1}$. As observed from Figure 5, the UTW-2TS-DNC protocol is not affected by the power allocation coefficients $\beta_{1}$ and $\beta_{2}$, and the UTW-2TS-SC protocol reaches the largest throughputs at approximate values $\beta_{1}=0.7$ and $\beta_{1}=0.8$ corresponding to perfect SICs and imperfect SICs, respectively. These values $\beta_{1}=0.7$ and $\beta_{1}=0.8$ are to equalize the SINR qualities between two hops from the selected secondary relay to the secondary sources.

Figure 6 shows the throughputs of the protocols UTW2TS-DNC and UTW-2TS-SC versus $\alpha_{1}$ and $\beta_{1}$ in the three- 

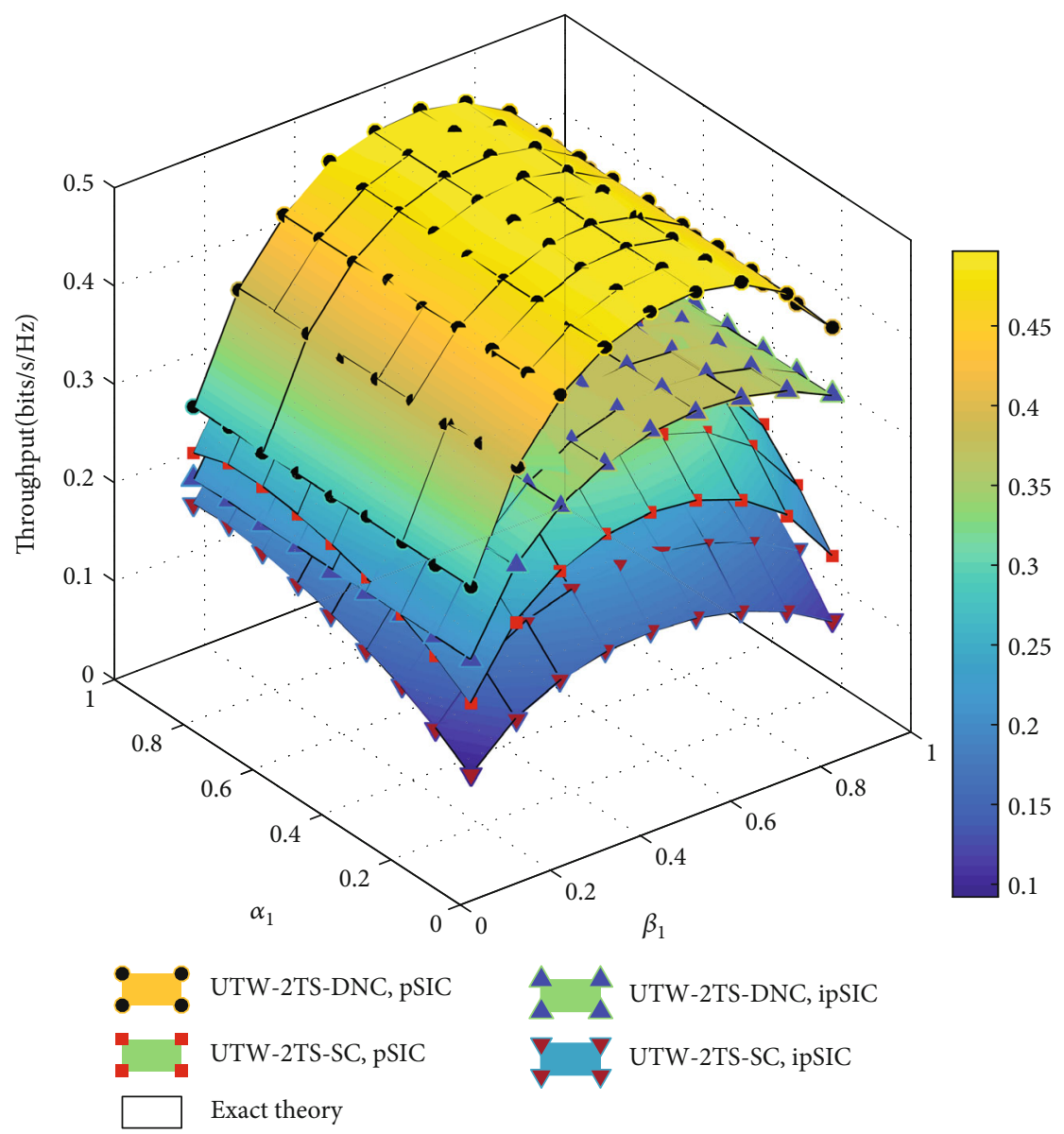

Figure 6: Throughputs of the protocols UTW-2TS-DNC and UTW-2TS-SC versus $\alpha_{1}$ and $\beta_{1}$ when $Q=10(\mathrm{~dB}), \Psi=-5(\mathrm{~dB}), x_{R}=0.3, y_{R}=0$ , $x_{\mathrm{PR}}=y_{\mathrm{PR}}=0.5, M=3, \alpha_{2}=1-\alpha_{1}$, and $\beta_{2}=1-\beta_{1}$.

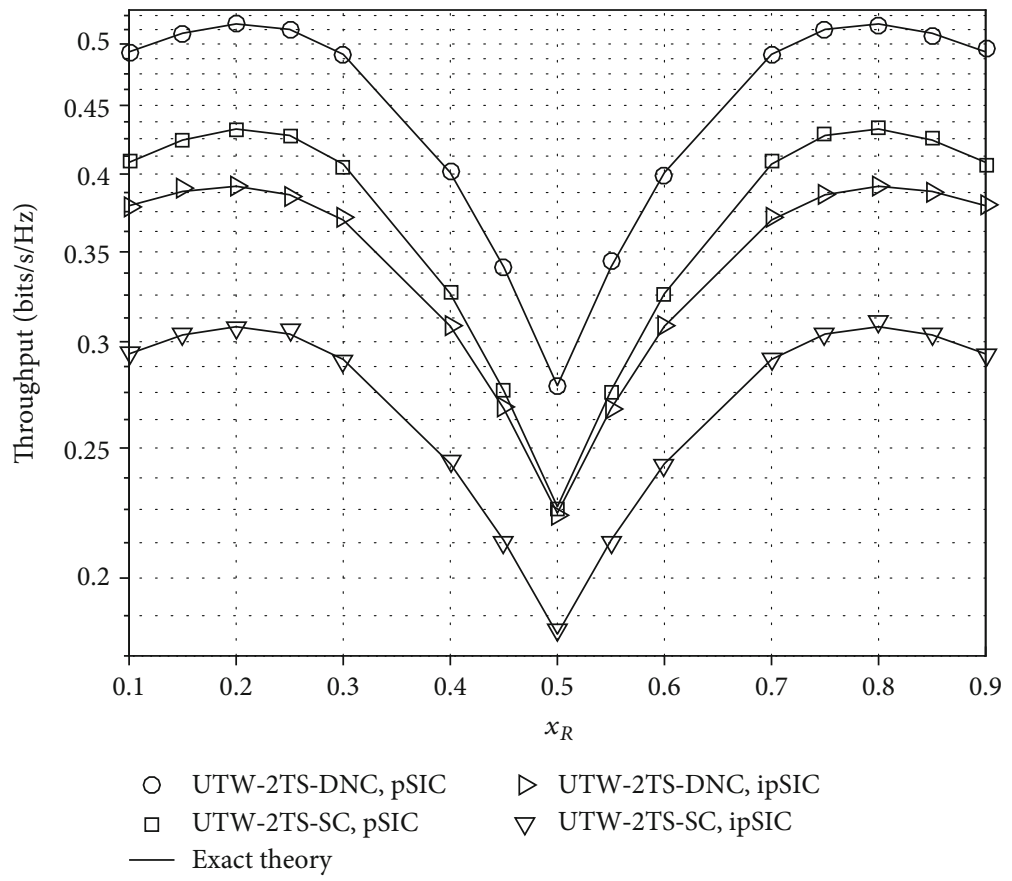

Figure 7: Throughputs of the protocols UTW-2TS-DNC and UTW-2TS-SC versus $x_{R}$ when $Q=10(\mathrm{~dB}), \alpha_{1}=\alpha_{2}=0.5, \beta_{1}=\beta_{2}=0.5, \Psi=-5$ $(\mathrm{dB}), x_{\mathrm{PR}}=y_{\mathrm{PR}}=0.5, M=3$, and $y_{\mathrm{R}}=0$. 


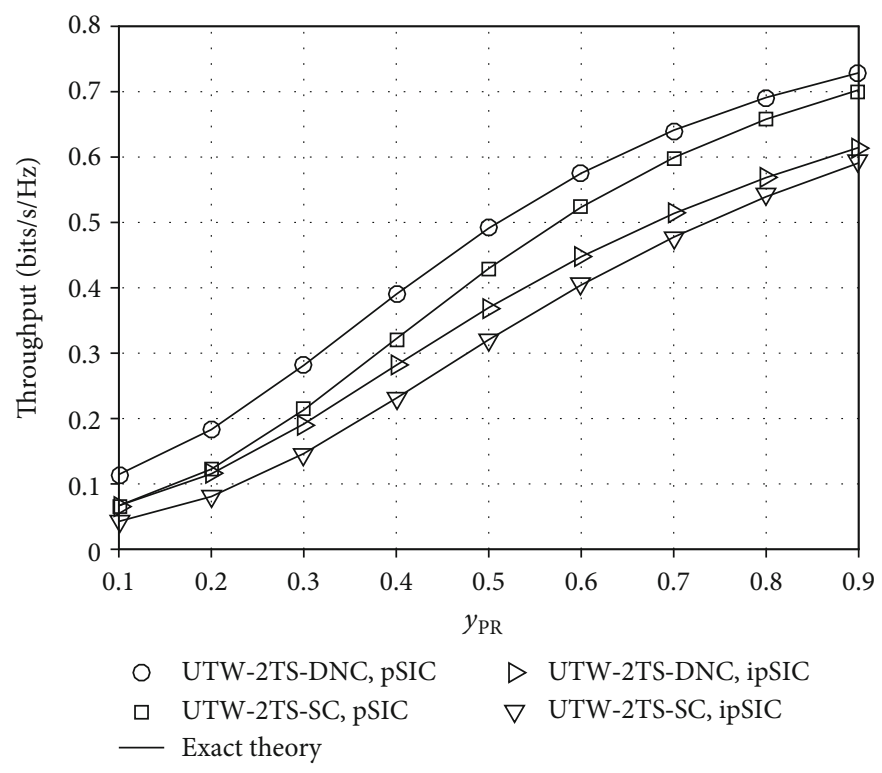

FIgURE 8: Throughputs of the protocols UTW-2TS-DNC and UTW-2TS-SC versus $y_{\mathrm{PR}}$ when $Q=10(\mathrm{~dB}), \alpha_{1}=\alpha_{2}=0.5, \beta_{1}=0.7, \beta_{2}=1-$ $\beta_{1}=0.3, \Psi=-5(\mathrm{~dB}), x_{R}=0.3, y_{R}=0, M=3$, and $x_{\mathrm{PR}}=0.5$.

dimensional plane when $Q=10(\mathrm{~dB}), \Psi=-5(\mathrm{~dB}), x_{R}=0.3$, $y_{R}=0, x_{\mathrm{PR}}=y_{\mathrm{PR}}=0.5, M=3$, and $\alpha_{2}$ and $\beta_{2}$ are set as $\alpha_{2}=$ $1-\alpha_{1}$ and $\beta_{2}=1-\beta_{1}$. The value ranges of $\alpha_{1}$ and $\beta_{1}$ are established between 0.1 and 0.9 . The results from Figure 6 confirm the contributions from Figures 4 and 5 .

Figure 7 presents the throughputs of the protocols UTW2TS-DNC and UTW-2TS-SC versus $x_{R}$ when $Q=10(\mathrm{~dB})$, $\alpha_{1}=\alpha_{2}=0.5, \beta_{1}=\beta_{2}=0.5, \Psi=-5(\mathrm{~dB}), x_{\mathrm{PR}}=y_{\mathrm{PR}}=0.5, M$ $=3$, and $y_{R}$ is fixed as $y_{R}=0$. In this simulation, the normalized distances $d_{4}$ and $d_{5}$ are fixed at the same value 0.7 and the remaining normalized distances $d_{1}, d_{2}$, and $d_{3}$ are changed from 0.1 to 0.9 for $d_{1}, 0.9$ to 0.1 for $d_{2}$ (the distance $d_{2}$ has a reverse change versus the distance $d_{1}$ ), and 0.64 to 0.5 and back to 0.64 for $d_{3}$. From Figure 7, these proposed protocols UTW-2TS-DNC and UTW-2TS-SC achieve the largest throughputs at asymmetric locations of the selected secondary relay as $x_{R} \approx 0.2\left(d_{1}=0.2, d_{2}=0.8\right)$ and $x_{R} \approx 0.8\left(d_{1}=\right.$ $\left.0.8, d_{2}=0.2\right)$ because of decoding the received data sequentially (SIC operations). In addition, at the symmetric location $x_{R}=0.5\left(d_{1}=d_{2}=0.5\right)$, the throughputs of both protocols again have the lowest values.

Figure 8 presents the throughputs of the protocols UTW2TS-DNC and UTW-2TS-SC versus $y_{\mathrm{PR}}$ when $Q=10(\mathrm{~dB})$, $\alpha_{1}=\alpha_{2}=0.5, \beta_{1}=0.7, \beta_{2}=1-\beta_{1}=0.3, \Psi=-5(\mathrm{~dB}), x_{R}=$ $0.3, y_{R}=0, M=3$, and $x_{\mathrm{PR}}$ is fixed at $x_{\mathrm{PR}}=0.5$. In this case, the normalized distances $d_{1}$ and $d_{2}$ are fixed to 0.3 and 0.7 , respectively, and the primary receiver $(\mathrm{PR})$ is moving farther the secondary network characterized by changing the normalized distances $d_{3}, d_{4}$, and $d_{5}$ from 0.22 to 0.92 for $d_{3}$ and from 0.51 to 1.03 for $d_{4}$ and $d_{5}$. As shown in Figure 8, the throughputs of the protocols UTW-2TS-DNC and UTW-2TS-SC are enhanced when the value of $y_{\mathrm{PR}}$ increases because of low influence of the interference constraint to the sources and relays in the secondary network. Therefore, the secondary sources and relays can transmit with the maxi-

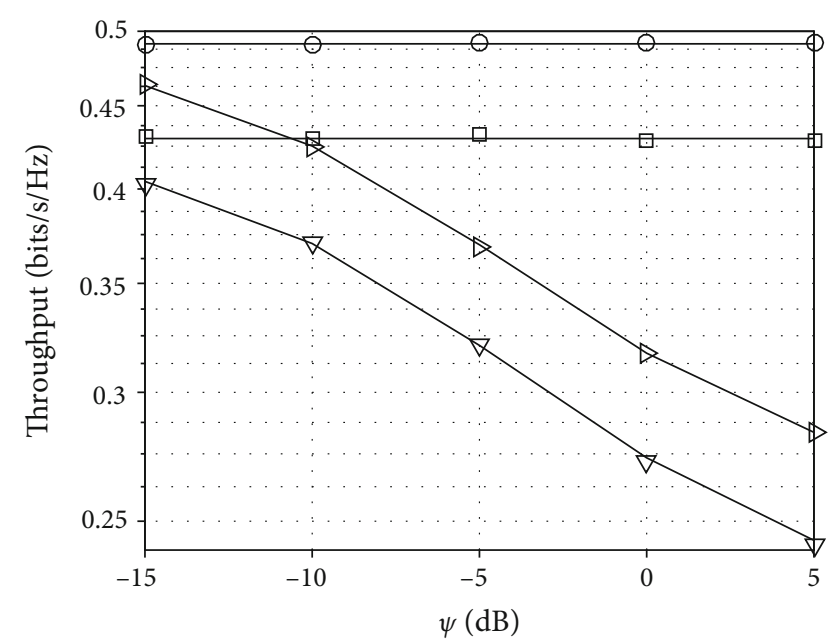

$\begin{array}{llll}\circ & \text { UTW-2TS-DNC, pSIC } & \triangleright & \text { UTW-2TS-DNC, ipSIC } \\ \square & \text { UTW-2TS-SC, pSIC } & \nabla & \text { UTW-2TS-SC, ipSIC } \\ & \text { Exact theory } & & \end{array}$

FIgURE 9: Throughputs of the protocols UTW-2TS-DNC and UTW-2TS-SC versus $\Psi$ when $Q=10(\mathrm{~dB}), \alpha_{1}=\alpha_{2}=0.5, \beta_{1}=0.7$, $\beta_{2}=1-\beta_{1}=0.3, x_{R}=0.3, y_{R}=0, x_{\mathrm{PR}}=y_{\mathrm{PR}}=0.5$, and $M=3$.

mum power, and interference cancelling and decoding capacity at the secondary relays and the secondary sources increase.

Figure 9 presents the throughputs of the protocols UTW2TS-DNC and UTW-2TS-SC versus the residual interference powers $\Psi$ when $Q=10(\mathrm{~dB}), \alpha_{1}=\alpha_{2}=0.5, \beta_{1}=0.7, \beta_{2}=1$ $-\beta_{1}=0.3, x_{R}=0.3, y_{R}=0, x_{\mathrm{PR}}=y_{\mathrm{PR}}=0.5$, and $M=3$. Considering the case of the imperfect SICs for both protocols, the throughput performances decrease when the residual interference powers increase. Furthermore, the throughputs of the protocols UTW-2TS-DNC and UTW-2TS-SC in the 
perfect SICs are upper limitations (expectations) of the ones in the imperfect SICs when $\Psi \rightarrow-\infty(\mathrm{dB})$.

\section{Conclusions}

In this paper, we proposed and analyzed the underlay twoway relaying scheme with two secondary sources and multiple secondary relays, known as the UTW-2TS scheme. The UTW-2TS scheme with the SIC solution operated in only two time slots and under an interference constraint of the primary receiver. The secondary relays decode successively the data transmitted by two secondary sources and then encode these data by two techniques: the DNC enforced by XOR operations (known as the UTW-2TS-DNC protocol) and the SC enforced by power domain additions (known as the UTW-2TS-SC protocol). A selected secondary relay which subjects to maximize the decoding capacities and to minimize the collection time of CSI in the proposed protocols UTW-2TS-DNC and UTW-2TS-SC suffered the residual interferences from the imperfect SIC operations. Exact outage probabilities and throughputs were derived to evaluate the system performance of the proposed UTW-2TSDNC and UTW-2TS-SC protocols. Simulation and analysis results provided discoveries of the performance improvements by increasing of the number of the cooperative secondary relays, the interference constraints, and the distances from the secondary network to the primary receiver. In addition, the proposed UTW-2TS-DNC and UTW-2TS-SC protocols achieved the best throughputs at optimal interference power allocation coefficients and optimal locations of the selected secondary relay. Considering the same power consumption, the UTW-2TS-DNC protocol performed better than the UTW-2TS-SC protocol. Finally, the analysis results of the outage probabilities and throughputs were validated by the Monte Carlo simulations.

\section{Appendix}

\section{A. Proof of Lemma 1}

Substituting (3) into the formula of $\Phi_{1}$ as in (18), we have the expression

$$
\begin{aligned}
\Phi_{1} & =\operatorname{Pr}\left\{\frac{\alpha_{1} Q g_{S_{1} R_{m}} g_{S_{2} \mathrm{PR}}}{\alpha_{2} Q g_{S_{2} R_{m}} g_{S_{1} \mathrm{PR}}+g_{S_{1} \mathrm{PR}} g_{S_{2} \mathrm{PR}}}<\gamma_{\mathrm{th}}\right\} \\
& =\operatorname{Pr}\left\{\frac{g_{S_{1} R_{m}}}{g_{S_{1} \mathrm{PR}}}<\frac{\gamma_{\mathrm{th}}\left(\alpha_{2} Q g_{S_{2} R_{m}}+g_{S_{2} \mathrm{PR}}\right)}{\alpha_{1} Q g_{S_{2} \mathrm{PR}}}\right\} \\
& =\operatorname{Pr}\{G_{S_{1} R_{m} / S_{1} \mathrm{PR}}<\underbrace{\frac{\gamma_{\mathrm{th}} \alpha_{2}}{\alpha_{1}}}_{v_{1}} G_{S_{2} R_{m} / S_{2} \mathrm{PR}}+\underbrace{\frac{\gamma_{\mathrm{th}}}{\alpha_{1} Q}}_{v_{1} Q}\} \\
& =\int_{0}^{\infty} f_{G_{S_{2} R_{m} / S_{2} \mathrm{PR}}}(y) \times F_{G_{S_{1} R_{m} / S_{1} \mathrm{PR}}}\left(v_{1} y+v_{2}\right) d y .
\end{aligned}
$$

From (23), we infer the CDF and the corresponding PDF of the RV $G_{S_{2} R_{m} / S_{2} \text { PR }}$ as

$$
\begin{aligned}
F_{G_{S_{2} R_{m} / S_{2} \mathrm{PR}}}(z) & =\lambda_{5} \sum_{p=0}^{1}\left(\begin{array}{l}
0 \\
1
\end{array}\right) \frac{(-1)^{p}}{\lambda_{5}+p \lambda_{2} z}=\lambda_{5}-\frac{1}{\lambda_{5}+\lambda_{2} z} \\
& =\frac{\lambda_{2} z}{\lambda_{5}+\lambda_{2} z}
\end{aligned}
$$

$$
f_{G_{S_{2} R_{m} / S_{2} \mathrm{PR}}}(z)=\frac{\partial F_{G_{S_{2} R_{m} / S_{2} \mathrm{PR}}}(z)}{\partial z}=\frac{\lambda_{2} \lambda_{5}}{\left(\lambda_{5}+\lambda_{2} z\right)^{2}} .
$$

Substituting (23) and (A.3) into (A.1), we have the equivalent formula:

$$
\begin{aligned}
\Phi_{1} & =\int_{0}^{\infty} \frac{\lambda_{2} \lambda_{5}}{\left(\lambda_{5}+\lambda_{2} y\right)^{2}} \lambda_{4} \sum_{p=0}^{M}\left(\begin{array}{c}
p \\
M
\end{array}\right) \frac{(-1)^{p}}{\lambda_{4}+p \lambda_{1}\left(v_{1} y+v_{2}\right)} d y \\
& =\lambda_{2} \lambda_{4} \lambda_{5} \sum_{p=0}^{M}\left(\begin{array}{c}
p \\
M
\end{array}\right)(-1)^{p} \int_{0}^{\infty} \frac{d y}{\left(\lambda_{4}+p \lambda_{1} v_{2}+p \lambda_{1} v_{1} y\right)\left(\lambda_{5}+\lambda_{2} y\right)^{2}} .
\end{aligned}
$$

By performing variable transformations for the integral in (A.4) as $t=1 /\left(\lambda_{5}+\lambda_{2} y\right)$ and $y=v_{4}(p)+\left(\lambda_{2} v_{3}(p)-\lambda_{5}\right.$ $\left.v_{4}(p)\right) t$, where $v_{3}(p)=\lambda_{4}+p \lambda_{1} v_{2}$ and $v_{4}(p)=p \lambda_{1} v_{1}$, Lemma 1 is proven completely.

\section{B. Proof of Lemma 2}

Substituting (3) and (6) into the formula of $\Phi_{2}$ as in (18), the probability $\Phi_{2}$ is expressed as

$$
\begin{aligned}
\Phi_{2} & =\operatorname{Pr}\left\{\left(\frac{\alpha_{1} Q g_{S_{1} R_{m}} g_{S_{2} \mathrm{PR}}}{\alpha_{2} Q g_{S_{2} R_{m}} g_{S_{1} \mathrm{PR}}+g_{S_{1} \mathrm{PR}} g_{S_{2} \mathrm{PR}}} \geq \gamma_{\text {th }}\right) \cap\left(\frac{P_{S_{2}} g_{S_{2} R_{m}}}{\varepsilon I\left|r_{m}\right|^{2}+\sigma^{2}}<\gamma_{\text {th }}\right)\right\} \\
& =\operatorname{Pr}\left\{\left(G_{S_{1} R_{m} / S_{1} \mathrm{PR}} \geq v_{1} G_{S_{2} R_{m} / S_{2} \mathrm{PR}}+v_{2}\right) \cap(G_{S_{2} R_{m} / S_{2} \mathrm{PR}}<\underbrace{\frac{\gamma_{\text {th }}}{\alpha_{2} Q}}_{v_{5}}+\underbrace{\left.\left.\varepsilon \frac{\gamma_{\text {th }}}{\alpha_{2}} g_{m}\right)\right\} .}_{v_{6}}\right.
\end{aligned}
$$

In (B.1), we consider two cases of perfect SICs $(\varepsilon=0)$ and imperfect SICs $(\varepsilon=1)$ to solve the following:

Case $\varepsilon=0$ : formula (B.1) is expressed and manipulated as

$$
\begin{aligned}
\Phi_{2} & =\operatorname{Pr}\left\{\left(G_{S_{1} R_{m} / S_{1} \mathrm{PR}} \geq v_{1} G_{S_{2} R_{m} / S_{2} \mathrm{PR}}+v_{2}\right) \cap\left(G_{S_{2} R_{m} / S_{2} \mathrm{PR}}<v_{5}\right)\right\} \\
& =\int_{0}^{v_{5}} f_{G_{S_{2} R_{m} / S_{2} \mathrm{PR}}}(y) \times\left(1-F_{G_{S_{1} R_{m} / S_{1} \mathrm{PR}}}\left(v_{1} y+v_{2}\right)\right) d y \\
& =F_{G_{S_{2} R_{m} / S_{2} \mathrm{PR}}}\left(v_{5}\right)-\int_{0}^{v_{5}} f_{G_{S_{2} R_{m} / S_{2} \mathrm{PR}}}(y) \times F_{G_{S_{1} R_{m} / S_{1} \mathrm{PR}}}\left(v_{1} y+v_{2}\right) d y,
\end{aligned}
$$

where $F_{G_{S_{2} R_{m} / S_{2} \mathrm{PR}}}\left(v_{5}\right)$ is the CDF of the RV $G_{S_{2} R_{m} / S_{2} \mathrm{PR}}$ at a value $v_{5}=\gamma_{\mathrm{th}} /\left(\alpha_{2} Q\right)$ (see (A.2)). 
By using the PDF of the RV $G_{S_{2} R_{m} / S_{2} \text { PR }}$ as in (A.3) and the $\mathrm{CDF}$ of the RV $G_{S_{1} R_{m} / S_{1} \mathrm{PR}}$ as in (23), formula (B.2) is rewritten as

$$
\begin{aligned}
\Phi_{2}= & F_{G_{S_{2} R_{m} S_{2} \mathrm{PR}}}\left(v_{5}\right)-\lambda_{2} \lambda_{4} \lambda_{5} \sum_{p=0}^{M}\left(\begin{array}{c}
p \\
M
\end{array}\right)(-1)^{p} \int_{0}^{v_{5}} \\
& \cdot \frac{d y}{\left(\lambda_{4}+p \lambda_{1} v_{2}+p \lambda_{1} v_{1} y\right)\left(\lambda_{5}+\lambda_{2} y\right)^{2}} .
\end{aligned}
$$

$$
\begin{aligned}
& \Phi_{2}=\int_{0}^{\infty} \Omega e^{-\Omega t}\left\{\frac{\lambda_{2}\left(v_{5}+v_{6} t\right)}{\lambda_{5}+\lambda_{2}\left(v_{5}+v_{6} t\right)}-\lambda_{2} \lambda_{4} \lambda_{5} \sum_{p=0}^{M}\left(\begin{array}{c}
p \\
M
\end{array}\right) \frac{(-1)^{p}}{\lambda_{2} v_{3}(p)-\lambda_{5} v_{4}(p)}\right. \\
& \left.\times\left(\frac{\lambda_{2}\left(v_{5}+v_{6} t\right)}{\lambda_{5}\left(\lambda_{5}+\lambda_{2}\left(v_{5}+v_{6} t\right)\right)}-\frac{v_{4}(p)}{\lambda_{2} v_{3}(p)-\lambda_{5} v_{4}(p)} \ln \left(\frac{\left(\lambda_{5}+\lambda_{2}\left(v_{5}+v_{6} t\right)\right) v_{3}(p)}{\lambda_{5}\left(v_{3}(p)+\left(v_{5}+v_{6} t\right) v_{4}(p)\right)}\right)\right)\right\} d t \\
& =\underbrace{\Omega \lambda_{2} \int_{0}^{\infty} \frac{\left(v_{5}+v_{6} t\right) e^{-\Omega t}}{\lambda_{5}+\lambda_{2}\left(v_{5}+v_{6} t\right)} d t}_{I_{1}}-\underbrace{\Omega \lambda_{2}{ }^{2} \lambda_{4} \sum_{p=0}^{M}\left(\begin{array}{c}
p \\
M
\end{array}\right) \frac{(-1)^{p}}{\lambda_{2} v_{3}(p)-\lambda_{5} v_{4}(p)} \int_{0}^{\infty} \frac{\left(v_{5}+v_{6} t\right) e^{-\Omega t}}{\lambda_{5}+\lambda_{2}\left(v_{5}+v_{6} t\right)} d t}_{I_{2}} \\
& +\underbrace{\Omega \lambda_{2} \lambda_{4} \lambda_{5} \sum_{p=0}^{M}\left(\begin{array}{c}
p \\
M
\end{array}\right) \frac{(-1)^{p} v_{4}(p)}{\left(\lambda_{2} v_{3}(p)-\lambda_{5} v_{4}(p)\right)^{2}} \int_{0}^{\infty} e^{-\Omega t} \ln \left(\frac{\left(\lambda_{5}+\lambda_{2}\left(v_{5}+v_{6} t\right)\right) v_{3}(p)}{\lambda_{5} \times\left(v_{3}(p)+\left(v_{5}+v_{6} t\right) v_{4}(p)\right)}\right) d t}_{I_{3}}
\end{aligned}
$$

We solve sequentially the integrals $I_{1}, I_{2}$, and $I_{3}$ in (B.5) as

$$
\begin{aligned}
& I_{1}=1-\frac{\Omega \lambda_{5}}{\lambda_{2} v_{6}} e^{\left(\lambda_{5}+\lambda_{2} v_{5}\right) \Omega /\left(\lambda_{2} v_{6}\right)} \Gamma\left(0, \frac{\left(\lambda_{5}+\lambda_{2} v_{5}\right) \Omega}{\lambda_{2} v_{6}}\right), \\
& I_{2}=\lambda_{2} \lambda_{4} \sum_{p=0}^{M}\left(\begin{array}{c}
p \\
M
\end{array}\right) \frac{(-1)^{p}}{\lambda_{2} v_{3}(p)-\lambda_{5} v_{4}(p)}-\Omega \lambda_{2} \lambda_{4} \lambda_{5} \sum_{p=0}^{M}\left(\begin{array}{c}
p \\
M
\end{array}\right) \\
& \cdot \frac{(-1)^{p}}{\lambda_{2} v_{3}(p)-\lambda_{5} v_{4}(p)} \int_{0}^{\infty} \frac{e^{-\Omega t}}{\lambda_{5}+\lambda_{2} v_{5}+\lambda_{2} v_{6} t} d t \\
& =\lambda_{2} \lambda_{4} \sum_{p=0}^{M}\left(\begin{array}{l}
p \\
M
\end{array}\right) \frac{(-1)^{p}}{\lambda_{2} v_{3}(p)-\lambda_{5} v_{4}(p)}-\frac{\Omega \lambda_{4} \lambda_{5}}{v_{6}} \sum_{p=0}^{M}\left(\begin{array}{c}
p \\
M
\end{array}\right) \\
& \cdot \frac{(-1)^{p} e^{\left(\lambda_{5}+\lambda_{2} v_{5}\right) \Omega /\left(\lambda_{2} v_{6}\right)}}{\lambda_{2} v_{3}(p)-\lambda_{5} v_{4}(p)} \Gamma\left(0, \frac{\left(\lambda_{5}+\lambda_{2} v_{5}\right) \Omega}{\lambda_{2} v_{6}}\right)
\end{aligned}
$$

$$
\begin{aligned}
I_{3}=\lambda_{2} & \lambda_{4} \lambda_{5} \sum_{p=0}^{M}\left(\begin{array}{c}
p \\
M
\end{array}\right) \frac{(-1)^{p} v_{4}(p)}{\left(\lambda_{2} v_{3}(p)-\lambda_{5} v_{4}(p)\right)^{2}} \\
& \cdot\left\{\ln \left(\frac{v_{3}(p)}{\lambda_{5}}\right)+\Omega \int_{0}^{\infty} e^{-\Omega t} \ln \left(\frac{\lambda_{5}+\lambda_{2}\left(v_{5}+v_{6} t\right)}{v_{3}(p)+\left(v_{5}+v_{6} t\right) v_{4}(p)}\right) d t\right\}
\end{aligned}
$$

$$
=\lambda_{2} \lambda_{4} \lambda_{5} \sum_{p=0}^{M}\left(\begin{array}{c}
p \\
M
\end{array}\right) \frac{(-1)^{p} v_{4}(p)}{\left(\lambda_{2} v_{3}(p)-\lambda_{5} v_{4}(p)\right)^{2}}
$$$$
\cdot\left\{\ln \left(\frac{v_{3}(p)}{\lambda_{5}}\right)+\Omega \int_{0}^{\infty} e^{-\Omega t} \ln \left(\lambda_{5}+\lambda_{2} v_{5}+\lambda_{2} v_{6} t\right) d t\right.
$$$$
\left.-\Omega \int_{0}^{\infty} e^{-\Omega t} \ln \left(v_{3}(p)+v_{5} v_{4}(p)+v_{6} v_{4}(p) t\right) d t\right\}
$$$$
=\lambda_{2} \lambda_{4} \lambda_{5} \sum_{p=0}^{M}\left(\begin{array}{l}
p \\
M
\end{array}\right) \frac{(-1)^{p} v_{4}(p)}{\left(\lambda_{2} v_{3}(p)-\lambda_{5} v_{4}(p)\right)^{2}}
$$$$
\cdot\left\{\ln \left(\frac{\left(\lambda_{5}+\lambda_{2} v_{5}\right) v_{3}(p)}{\lambda_{5}\left(v_{3}(p)+v_{5} v_{4}(p)\right)}\right)+e^{\left(\lambda_{5}+\lambda_{2} v_{5}\right) \Omega / \lambda_{2} v_{6}} \Gamma\right.
$$$$
\cdot\left(0, \frac{\left(\lambda_{5}+\lambda_{2} v_{5}\right) \Omega}{\lambda_{2} v_{6}}\right)-e^{\left(v_{3}(p)+v_{5} v_{4}(p)\right) \Omega / v_{6} v_{4}(p)} \Gamma
$$$$
\left.\cdot\left(0, \frac{\left(v_{3}(p)+v_{5} v_{4}(p)\right) \Omega}{v_{6} v_{4}(p)}\right)\right\}
$$ 
where $\Gamma(u, v)$ is the upper incomplete Gamma function ([42], eq. 8.350.2).

Substituting (B.6), (B.7), and (B.8) into (B.5), the probability $\Phi_{2}$ is analyzed for the case $\varepsilon=1$ as (26) in Lemma 2 . Hence, the lemma is proven completely.

\section{Data Availability}

The data used to support the findings of this study are included within the article.

\section{Conflicts of Interest}

The authors declare that there is no conflict of interest regarding the publication of this paper.

\section{Acknowledgments}

This research is funded by Vietnam National Foundation for Science and Technology Development (NAFOSTED) under grant number 102.04-2019.13. Khuong Ho-Van acknowledges the support of time and facilities from Ho Chi Minh City University of Technology (HCMUT), VNU-HCM, for this study.

\section{References}

[1] I. F. Akyildiz, W.-Y. Lee, M. C. Vuran, and S. Mohanty, "NeXt generation/dynamic spectrum access/cognitive radio wireless networks: a survey," Computer Networks, vol. 50, no. 13, pp. 2127-2159, 2006.

[2] J. Mitola and G. Q. Maguire, "Cognitive radio: making software radios more personal," IEEE Personal Communications, vol. 6, no. 4, pp. 13-18, 1999.

[3] A. Goldsmith, S. A. Jafar, I. Maric, and S. Srinivasa, "Breaking spectrum gridlock with cognitive radios: an information theoretic perspective," Proceedings of the IEEE, vol. 97, no. 5, pp. 894-914, 2009.

[4] H. B. Ahmad, "Ensemble classifier based spectrum sensing in cognitive radio networks," Wireless Communications and Mobile Computing, vol. 2019, Article ID 9250562, 16 pages, 2019.

[5] Y. J. Chun, M. O. Hasna, and A. Ghrayeb, "Adaptive network coding for spectrum sharing systems," IEEE Transactions on Wireless Communications, vol. 14, no. 2, pp. 639-654, 2014.

[6] Y. He, J. Evans, and S. Dey, "Secrecy rate maximization for cooperative overlay cognitive radio networks with artificial noise," in 2014 IEEE International Conference on Communications (ICC), pp. 1663-1668, Sydney, NSW, Australia, June 2014.

[7] P. N. Son, "Joint impacts of hardware impairments, imperfect CSIs, and interference constraints on underlay cooperative cognitive networks with reactive relay selection," Telecommunication Systems, vol. 71, no. 1, pp. 65-76, 2019.

[8] T. M. C. Chu and H. J. Zepernick, "Performance optimization for hybrid two-way cognitive cooperative radio networks with imperfect spectrum sensing," IEEE Access, vol. 6, pp. 7058270596, 2018.

[9] K. Ho-Van and T. Do-Dac, "Security analysis for underlay cognitive network with energy-scavenging capable relay over Nakagami-M fading channels," Wireless Communications and Mobile Computing, vol. 2019, Article ID 5080952, 16 pages, 2019.

[10] P. Popovski and H. Yomo, "Physical network coding in twoway wireless relay channels," in 2007 IEEE International Conference on Communications, Glasgow, UK, June 2007.

[11] Y. Li, R. H. Y. Louie, and B. Vucetic, "Relay selection with network coding in two-way relay channels," IEEE Transactions on Vehicular Technology, vol. 59, no. 9, pp. 44894499, 2010.

[12] T. T. Duy and H. Y. Kong, "Exact outage probability of cognitive two-way relaying scheme with opportunistic relay selection under interference constraint," IET Communications, vol. 6, no. 16, pp. 2750-2759, 2012.

[13] H. V. Toan and V. N. Q. Bao, "Opportunistic relaying for cognitive two-way network with multiple primary receivers over Nakagami-m fading," in 2016 International Conference on Advanced Technologies for Communications (ATC), Hanoi, Vietnam, October 2016

[14] H. V. Toan, V. N. Quoc Bao, and H. Nguyen-le, "Cognitive two-way relay systems with multiple primary receivers: exact and asymptotic outage formulation," IET Communications, vol. 11, no. 16, pp. 2490-2497, 2017.

[15] S. Hatamnia, S. Vahidian, S. Aïssa, B. Champagne, and M. Ahmadian-Attari, "Network-coded two-way relaying in spectrum-sharing systems with quality-of-service requirements," IEEE Transactions on Vehicular Technology, vol. 66, no. 2, pp. 1299-1312, 2017.

[16] H. V. Toan, V. N. Q. Bao, and K. N. Le, "Performance analysis of cognitive underlay two-way relay networks with interference and imperfect channel state information," EURASIP Journal on Wireless Communications and Networking, vol. 2018, no. 1, 2018.

[17] S. Solanki, P. K. Sharma, and P. K. Upadhyay, "Adaptive link utilization in two-way spectrum sharing relay systems under average interference-constraints," IEEE Systems Journal, vol. 12, no. 4 , pp. 3461-3472, 2018.

[18] Z. Cao, X. Ji, J. Wang, S. Zhang, Y. Ji, and J. Wang, "Securityreliability tradeoff analysis for underlay cognitive two-way relay networks," IEEE Transactions on Wireless Communications, vol. 18, no. 12, pp. 6030-6042, 2019.

[19] X. Yue, Y. Liu, S. Kang, A. Nallanathan, and Y. Chen, "Modeling and analysis of two-way relay non-orthogonal multiple access systems," IEEE Transactions on Communications, vol. 66, no. 9, pp. 3784-3796, 2018.

[20] X. Zou, B. He, and H. Jafarkhani, “An analysis of two-user uplink asynchronous non-orthogonal multiple access systems," IEEE Transactions on Wireless Communications, vol. 18, no. 2, pp. 1404-1418, 2019.

[21] Z. Yang, Z. Ding, P. Fan, and N. Al-Dhahir, "The impact of power allocation on cooperative non-orthogonal multiple access networks with SWIPT," IEEE Transactions on Wireless Communications, vol. 16, no. 7, pp. 4332-4343, 2017.

[22] A. Agarwal and A. K. Jagannatham, "Performance analysis for non-orthogonal multiple access (NOMA)-based two-way relay communication," IET Communications, vol. 13, no. 4, pp. 363-370, 2019.

[23] P. N. Son and T. T. Duy, "A new approach for two-way relaying networks: improving performance by successive interference cancellation, digital network coding and opportunistic relay selection," Wireless Networks, vol. 26, no. 2, pp. 1315-1329, 2020. 
[24] X. Ding, T. Song, Y. Zou, X. Chen, and L. Hanzo, "Securityreliability tradeoff analysis of artificial noise aided two-way opportunistic relay selection," IEEE Transactions on Vehicular Technology, vol. 66, no. 5, pp. 3930-3941, 2017.

[25] P. N. Son and H. Y. Kong, "Exact outage analysis of energy harvesting underlay cooperative cognitive networks," IEICE Transactions on Communications, vol. E98.B, no. 4, pp. 661672, 2015.

[26] K. Tourki, K. A. Qaraqe, and M. S. Alouini, "Outage analysis for underlay cognitive networks using incremental regenerative relaying," IEEE Transactions on Vehicular Technology, vol. 62, no. 2, pp. 721-734, 2013.

[27] J. N. Laneman, D. N. C. Tse, and G. W. Wornell, "Cooperative diversity in wireless networks: efficient protocols and outage behavior," IEEE Transactions on Information Theory, vol. 50, no. 12, pp. 3062-3080, 2004.

[28] A. Papoulis and S. U. Pillai, Probability, Random Variables and Stochastic Processes, McGraw-Hill, New York, NY, USA, 4th edition, 2002.

[29] H. Kim, S. Lim, H. Wang, and D. Hong, "Optimal power allocation and outage analysis for cognitive full duplex relay systems," IEEE Transactions on Wireless Communications, vol. 11, no. 10, pp. 3754-3765, 2012.

[30] P. N. Son and T. T. Duy, "Performance analysis of underlay cooperative cognitive full-duplex networks with energyharvesting relay," Computer Communications, vol. 122, pp. 9-19, 2018.

[31] T. V. Nguyen, T. N. Do, V. N. Q. Bao, D. B. . Costa, and B. An, "On the performance of multihop cognitive wireless powered D2D communications in WSNs," IEEE Transactions on Vehicular Technology, vol. 69, no. 3, pp. 2684-2699, 2020.

[32] Y. Ruan, Y. Li, C. X. Wang, R. Zhang, and H. Zhang, "Energy efficient power allocation for delay constrained cognitive satellite terrestrial networks under interference constraints," IEEE Transactions on Wireless Communications, vol. 18, no. 10, pp. 4957-4969, 2019.

[33] T. V. Nguyen and B. An, "Cognitive multihop wireless powered relaying networks over Nakagami- $m$ fading channels," IEEE Access, vol. 7, pp. 154600-154616, 2019.

[34] P. Chakraborty and S. Prakriya, "Securing primary downlink transmissions from untrusted receivers of co-existing underlay cognitive networks," IEEE Transactions on Cognitive Communications and Networking, vol. 5, no. 3, pp. 491-503, 2019.

[35] H. Arezumand, H. Zamiri-Jafarian, and E. Soleimani-Nasab, "Exact and asymptotic analysis of partial relay selection for cognitive RF-FSO systems with non-zero boresight pointing errors," IEEE Access, vol. 7, pp. 58611-58625, 2019.

[36] H. Gao, S. Zhang, Y. Su, M. Diao, and M. Jo, "Joint multiple relay selection and time slot allocation algorithm for the EHabled cognitive multi-user relay networks," IEEE Access, vol. 7, pp. 111993-112007, 2019.

[37] L. Pei, T. Zhifeng, L. Zinan, E. Erkip, and S. Panwar, "Cooperative wireless communications: a cross-layer approach," IEEE Wireless Communications, vol. 13, no. 4, pp. 84-92, 2006.

[38] M. Matthaiou, A. Papadogiannis, E. Bjornson, and M. Debbah, "Two-way relaying under the presence of relay transceiver hardware impairments," IEEE Communications Letters, vol. 17, no. 6, pp. 1136-1139, 2013.

[39] P. N. Son, D. Har, and H. Y. Kong, "Smart power allocation for secrecy transmission in reciprocally cooperative spectrum sharing," IEEE Transactions on Vehicular Technology, vol. 64, no. 11, pp. 5395-5400, 2015.

[40] A. A. Nasir, X. Zhou, S. Durrani, and R. A. Kennedy, "Relaying protocols for wireless energy harvesting and information processing," IEEE Transactions on Wireless Communications, vol. 12, no. 7, pp. 3622-3636, 2013.

[41] P. K. Sharma and P. K. Upadhyay, "Performance analysis of cooperative spectrum sharing with multiuser two-way relaying over fading channels," IEEE Transactions on Vehicular Technology, vol. 66, no. 2, pp. 1324-1333, 2017.

[42] R. I. Gradshteyn, I. S. Jeffrey, and A. D. Zwillinger, Table of Integral, Series and Products, Elsevier, Amsterdam, 7th edition, 2007. 